\title{
Angiotensin II disrupts the cytoskeletal architecture of human urine-derived podocytes and results in activation of the renin-angiotensin system
}

\author{
Lars Erichsen ${ }^{1}$, Martina Bohndorf ${ }^{1}$, Md. Shaifur Rahman ${ }^{1}$, Wasco Wruck ${ }^{1}$ and James Adjaye ${ }^{1^{*}}$
}

\begin{abstract}
High blood pressure is one of the major public health problems which causes severe disorders in several tissues including the human kidney. One of the most important signaling pathways associated with the regulation of blood pressure is the renin-angiotensin system (RAS), with its main mediator angiotensin II (ANGII). Elevated levels of circulating and intracellular ANGII and aldosterone lead to pro-fibrotic, -inflammatory and -hypertrophic milieu that causes remodelling and dysfunction in cardiovascular and renal tissues. Furthermore, ANGII has been recognized as major risk factor for the induction of apoptosis in podocytes, ultimately leading to chronic kidney disease (CDK).

In the past, disease modeling of kidney-associated malignancies was extremely difficult, as the derivation of kidney originated cells is very challenging. Here we describe a differentiation protocol for reproducible differentiation of SIX2-positive urine derived renal progenitor cells (UdRPCS) into mature podocytes bearing typical foot processes. The UdRPCs-derived podocytes show the ability to execute Albumin endocytosis and the activation of the renin-angiotensin system by being responsive to ANGII stimulation. Our data reveals the ANGII dependent downregulation of NPHS1 and SYNPO, resulting in the disruption of the complex podocyte cytoskeletal architecture, as shown by immunofluorescence-based detection of $\alpha$-ACTININ. In the present manuscript we confirm and propose UdRPCs as a unique cell type useful for studying nephrogenesis and associated diseases. Furthermore, the responsiveness of UdRPCs-derived podocytes to ANGII implies potential applications in nephrotoxicity studies and drug screening.
\end{abstract}

${ }^{1}$ Institute for Stem Cell Research and Regenerative Medicine, Medical Faculty, Heinrich-HeineUniversity Düsseldorf, 40225 Düsseldorf, Germany

\section{*Correspondence}

E-mail: james.adjaye@med.uni-duesseldorf.de

Keywords: renal differentiation, podocytes, disease modeling, renin-angiotensin-system 


\section{Introduction}

The kidney glomerulus or renal corpuscle consists of a glomerular tuft and the Bowman's capsule. Its major task is the filtration of blood to generate urine. The glomerulus consists of distinct cell types: endothelial cells, mesangial cells, parietal epithelial cells of Bowman's capsule and podocytes, which are attached to the outer part of the glomerular basement membrane (GBM). Podocytes are pericyte-like cells with a complex cellular organization. Key characteristics are the cell body with major and minor foot processes (FPs) [1]. The FPs contain a highly organized actin-based cytoskeleton, essential for maintaining the complex architecture typical of podocytes. Alpha-actinin-4 (ACTN4) and Synaptopodin (SYNPO) are both highly expressed in podocyte foot processes and function as cross-linkers of F-actin filaments in order to bundle them and thereby enhancing podocyte signaling and mobility [2,3]. Filtration slits are formed by the spatial arrangement of FPs of neighboring podocytes and each of these slits is bridged by the so called glomerular slit diaphragm (SD), which is a comparable structure to the adherens junctions [1]. The most abundant proteins that contribute to SD formation are Nephrin (NPHS1) [4] and Podocin (NPHS2) [5]. Furthermore, Nephrin is also associated with the actin cytoskeleton thereby contributing to podocyte actin dynamics and FPs formation [6]. Together the FPs and SD establish the filtration barrier of the kidney with its selective permeability.

High blood pressure is one of the major public health problems, causing severe disorders in several tissues including heart, brain and kidney [7]. One of the most important signaling pathways in blood pressure regulation is the renin-angiotensin system (RAS), with its main mediator angiotensin II (ANGII). At the molecular level, ANGII signaling is mediated by two classes of receptors (AGTR1 and AGTR2). Both receptors are expressed in a wide variety of tissues (including the heart, kidney, blood vessels, adrenal glands and cardiovascular control centers in the brain) and upon stimulation control vasoconstriction $[8,9]$. Human Podocytes express both types of ANGII receptors and are indeed effector cells for this peptide [10]. Furthermore, elevated levels of ANGII have been identified as a main risk factor for the initiation and progression of chronic kidney disease (CKD). Increased ANGII concentrations are associated with the downregulation of Nephrin and Synaptopodin expression in podocytes [11, 12]. The depletion of NPHS1 and SNYPO is causative for podocyte injury [13], which is typically associated with marked albuminuria [1], and increased podocyte apoptosis [14]. This is especially crucial since mature podocytes are terminal differentiated cells that are unable to undergo cell division in vivo [1]. As a result of this, the replenishment capabilities of podocytes are limited and expose the glomerulus vulnerable to exogenous noxae. Long lasting hazardous cues, such as high blood pressure, can manifest in the significant loss of podocytes, which is a hallmark in the development of CKD [15]. 
In the past disease modeling for kidney-associated malignancies was extremely difficult, as the derivation of kidney originated cells is very challenging. This is particularly true for podocytes, since their complex architecture is not well preserved from kidney biopsy tissue [16]. To model podocyterelated diseases, researchers either used an immortalized podocyte cell line [16-18] or iPSC-based models [19-23]. Whilst the first approach shows the typical drawback of immortalized cells, namely chromosomal aneuploidies, thus making these cells prone to cancerous transformation. The iPSCbased differentiation approach lacks reproducibility and attainment of mature podocytes with elaborate foot processes.

We recently reported human urine as a non-invasive source of renal stem cells with regenerative potential [24]. The urine derived renal progenitor cells (UdRPCs) express renal stem cell markers such as SIX2, CITED1 WT1, CD133, CD24 and CD106. Stimulation of UdRPCs with the GSK3ß-inhibitor (CHIR99021) induced differentiation into renal epithelial proximal tubular cells. In the present study, we provide a detailed protocol for the direct differentiation of SIX2-positive UdRPCs into mature podocytes with typical foot processes without the need of immortalized cells or pluripotent stem cells. We provide the full characterization of the generated podocytes at the transcriptome, secretome and cellular level. Furthermore, with ANGII treatment we demonstrate the responsiveness of the podocytes to the renin-angiotensin system (RAS) with a downregulated expression of NPHS1 and SYNPO.

Our data demonstrates and establishes human urine-derived podocytes as a valuable in vitro model for studying podocyte injury, loss and ultimately CKD. In addition, we hypothesize that the cells can also be used for further drug testing and eventually kidney-associated regenerative therapies. 


\section{Material and Methods}

\section{Cell culture conditions}

UdRPCs were cultured in Proliferation Medium (PM) composed of 50\% DMEM high Glucose and 50\% Keratinocyte medium supplemented with 5\% FCS, 0.5\% NEAA, 0.25\% Gtx and 0.5\% Penicillin and Streptomycin at $37^{\circ} \mathrm{C}$ (Gibco, Carlsbad, United States) under hypoxic conditions. For differentiation, the cells were seeded at low and high density in 6 - or 12 -well plates coated with $0,2 \%$ type 1 Collagen (Gibco) and grown in PM for 24 hours. After this the medium was changed to advanced RPMI (Gibco) supplemented with $0.5 \%$ FCS, $1 \%$ Penicillin and Streptomycin and $30 \mu \mathrm{M}$ retinoic acid. Typical podocyte morphology was observed after 7 days. ANG II (Sigma Aldrich, St. Louis, Missouri, United States) was diluted in the culture medium to a final concentration of $100 \mu \mathrm{M}$. Cells were incubated for $6 \mathrm{~h}$ and $24 \mathrm{~h}$ with ANGII and the conditioned medium was kept for secretome analyses.

\section{Relative Quantification of podocyte associated gene expression by real-time PCR.}

Real time PCR of podocyte associated gene expression was performed as follows:

Real time measurements were carried out on the Step One Plus Real Time PCR Systems using MicroAmp Fast optical 384 Well Reaction Plate and Power Sybr Green PCR Master Mix (Applied Biosystems, Foster City, United States). The amplification conditions were denaturation at $95^{\circ} \mathrm{C}$ for $13 \mathrm{~min}$. followed by $37 \mathrm{cycles}$ of $95^{\circ} \mathrm{C}$ for $50 \mathrm{~s}, 60^{\circ} \mathrm{C}$ for $45 \mathrm{~s}$ and $72^{\circ} \mathrm{C}$ for $30 \mathrm{~s}$. Primer sequences are listed in supplement table 1.

\section{Bisulfite genomic sequencing}

Bisulfite sequencing was performed following bisulfite conversion with the EpiTec Kit (Qiagen, Hilden, Germany) as described [25]. Primers were designed after excluding pseudogenes or other closely related genomic sequences which could interfere with specific amplification by amplicon and primer sequences comparison in BLAT sequence data base (https://genome.ucsc.edu/FAQ/FAQblat.html). PCR primers are listed in supplementary table 2.

Briefly, the amplification conditions were denaturation at $95^{\circ} \mathrm{C}$ for $13 \mathrm{~min}$. followed by $37 \mathrm{cycles}$ of $95^{\circ} \mathrm{C}$ for $50 \mathrm{~s}, 54^{\circ} \mathrm{C}$ for $45 \mathrm{~s}$ and $72^{\circ} \mathrm{C}$ for $30 \mathrm{~s}$. The amplification product is $270 \mathrm{bp}$ in size. Amplification products were cloned into PCR2.1vector using the TA Cloning Kit (Invitrogen, Carlsbad, United States) according to the manufacturer's instructions. On average 30 clones were sequenced using the BigDye Terminator Cycle Sequencing Kit (Applied Biosystems) on a DNA analyzer 3700 (Applied Biosystems) with M13 primer to obtain a representative methylation profile of each sample. $5^{\prime}$-regulatory gene sequences are referring to +1 transcription start of the following sequence: 
Homo sapiens WT1 transcription factor (WT1), RefSeqGene (LRG_525) on chromosome 11

NCBI Reference Sequence: NG_009272.1

Homo sapiens NPHS2, podocin (NPHS2), RefSeqGene (LRG_887) on chromosome 1

NCBI Reference Sequence: NG_007535.1

\section{Immunofluorescence staining}

Cells were fixed with $4 \%$ paraformaldehyde (PFA) (Polysciences, Warrington, United States). To block unspecific binding sites the fixed cells were incubated with blocking buffer containing $10 \%$ normal goat or donkey serum, $1 \%$ BSA, $0.5 \%$ Triton, and $0.05 \%$ Tween, for $2 \mathrm{~h}$ at room temperature. Incubation of the primary antibody was performed at $4^{\circ} \mathrm{C}$ overnight in staining buffer (blocking buffer diluted 1:1 with PBS). After at least $16 \mathrm{~h}$ of incubation the cells were washed three times with PBS/0.05\% Tween and incubated with a 1:500 dilution of secondary antibodies dilution. Afterwards the cells were washed again three times with PBS/0.05\% Tween and nuclei were stained with Hoechst 1:5000 (Thermo Fisher Scientific, Waltham, United States) and podocyte cytoskeleton was stained with Alexa Flour 488 phalloidin (Thermo Fisher Scientific) (1:400). Images were captured using a fluorescence microscope (LSM700; Zeiss, Oberkochen, Germany) with Zenblue software (Zeiss). Individual channel images were processed and merged with Fiji. Detailed Information of the used antibodies are given in supplementary table 3.

\section{Microarray data analyses}

Total RNA $(1 \mu \mathrm{g})$ preparations were hybridized on the PrimeView Human Gene Expression Array (Affymetrix, Thermo Fisher Scientific, USA) at the core facility Biomedizinisches Forschungszentrum (BMFZ) of the Heinrich Heine University Düsseldorf. The raw data was imported into the R/Bioconductor environment and further processed with the package affy using backgroundcorrection, logarithmic (base 2) transformation and normalization with the Robust Multi-array Average (RMA) method. The heatmap.2 function from the gplots package was applied for cluster analysis and to generate heatmaps using Pearson correlation as similarity measure. Gene expression was detected using a detection-p-value threshold of 0.05. Differential gene expression was determined via the $p$-value from the limma package which was adjusted for false discovery rate using the q value package. Thresholds of 1.33 and 0.75 were used for up-/down-regulation of ratios and 0.05 for $p$-values. Venn diagrams were generated with the Venn Diagram package. Subsets from the venn diagrams were used for follow-up GO and pathway analyses as described by Zhou et al [26]. Gene expression data will be available online at the National Centre of Biotechnology Information (NCBI) Gene Expression Omnibus. 


\section{Secretome analyses}

Conditioned media from control podocytes as well as ANG II-treated cells were analysed using the Proteome Profiler Human Kidney Biomarker Array Kit distributed by Research And Diagnostic Systems, Inc. (Minneapolis, Minnesota, United States) as described by the manufacturer. Obtained images were analyzed by using the Image J software [27] with the Microarray Profile plugin by Bob Dougherty and Wayne Rasband (https://www.optinav.info/MicroArray_Profile.html). The integrated density generated by the Microarray profile plugin function Measure RT was used for follow-up processing which was performed in the R/Bioconductor environment [28]. Arrays were normalized employing the Robust Spline Normalization from the Bioconductor lumi package [29]. A threshold for background intensities was defined at $5 \%$ of the range between maximum and minimum intensity and a detection-p-value was calculated according to the method described in Graffmann et al. [30].

\section{Cluster analysis of the Renin-angiotensin pathway}

Pathways and associated genes were downloaded from the KEGG database [31] in July 2020 and annotated with official gene symbols. Genes from the KEGG pathway hsa04614 Renin-angiotensin system were extracted from the microarray data normalized with the RMA method from the package oligo [32] in R/Biocondcutor [28] as described above and subjected to the R heatmap() function using Pearson correlation as similarity measure and color scaling per row.

\section{Albumin endocytosis assay}

The functionality of podocyte differentiated from urine-derived renal progenitor cells was analyzed employing the Albumin endocytosis assay. In brief, podocytes were plated at low density of $10 \%$ in a 12-well plate coated with 0,2\% type 1 collagen. 48 hrs later the cells were washed with 1x PBS and replaced with podocyte culture medium supplemented with $20 \mu \mathrm{g} / \mathrm{ml}$ of BSA-Alexa Fluor ${ }^{\mathrm{TM}} 488$ and incubated at $37^{\circ} \mathrm{C}$ for 60 minutes. After incubation, the cells were washed $3 X$ with cold PBS and fixed with 4\% PFA for 15 minutes. Finally, the images were taken at the excitation wavelength of $488 \mathrm{~nm}$ and an emission wavelength of $540 \mathrm{~nm}$ using a LSM700 florescence microscope. 


\section{Results}

\section{Generation of human podocytes from urine derived renal progenitor cells}

We recently reported a "rice grain" fibroblast-like morphology resembling MSCs isolated directly from urine samples of 4 male (UM) and 6 female (UF) donors. These cells express the renal stem cell markers SIX2, CITED1 WT1, CD133, CD24 and CD106, we referred to these cells as urine derived renal progenitor cells (UdRPCs) [24]. UdRPCs maintain their stem cell features for almost 12 passages (fig. 1a). So far, we have established a differentiation protocol leading to renal epithelial proximal tubular lineage by supplementation with the GSK3ß-inhibitor (CHIR99021) or to podocytes as described in fig. 1a. UdRPCs acquire typical podocyte "fried egg" shape morphology after 7 days, when cultured at $70 \%$ confluency in adv. RPMI medium supplemented with retinoic acid. This protocol was applied to cells isolated from human urine of several individuals (age ranged between 45 and 76 years), resulting in the robust generation of podocytes (fig. 1a). For further analysis we evaluated mRNA expression of the two most abundant proteins within the FPs and SD of human podocytes, Nephrin (NPHS1) and Synaptopodin (SYNPO) respectively by quantitative real time PCR. We analysed mRNA expression of podocytes derived from three individuals, two males (UM48 and UM51) and a female (UF45) and compared it to their undifferentiated counterparts. The expression of NPHS1 and SYNPO was upregulated in the differentiated podocytes compared to the respective UdRPCs (fig. $1 b+c)$. To show methylation changes within the 5'-regulatory region of the key podocyte transcription factor WT1, we applied bisulfite genomic sequencing. In total a 270 bp long WT1 promoter fragment spanning 19 CpG-dinucleotides was analysed. We found, that the undifferentiated UdRPCs UM51 had a dense methylation pattern at CpG position 12 and 13 of the 5'-regulatory region (fig. 1d). Upon applying our differentiation protocol, methylated DNA at the respective positions for UM51-derived podocytes, was found to be almost completely lost (fig. 1e). As a mark of renal cell functionality, Albumin endocytosis was observed after exogenous BSA was supplemented to the culture medium (fig. 1f).

\section{Retinoic acid enhances podocyte differentiation}

To improve the differentiation protocol of UdRPCs into podocytes, we supplemented the adv. RPMI medium with $30 \mu \mathrm{M}$ retinoic acid and analysed the derived UM51-podocytes by immunofluorescence- based detection of expression of Nephrin (NPHS1), Podocin (NPHS2) and CD2AP (fig. 2a-f). While the UM51 podocytes cultured in adv. RPMI already show expression of all three proteins, which are necessary for podocyte functionality, the addition of retinoic acid significantly increased the protein expression levels of these key podocyte markers. Additionally, we measured the expression of $L M X 1 b, N P H S 1$, SYNPO and WT1 by quantitative real time PCR (fig. 2g-j). 
While the addition of retinoic acid had only minor impact on the expression of $L M X 1 b$ and $W T 1$, the expression levels of NPHS1 and SYNPO were highly increased, by 3 and 2-fold, respectively.

\section{Comparative transcriptome analysis of Urine derived Renal Progenitor cells with their podocyte counterparts}

After the successful and reproducible differentiation of UdRPCs into podocytes we performed a comparative transcriptome analysis of UF45, UM48 and UM51. Hierarchical clustering analysis comparing the transcriptomes of UdRPCs with podocytes revealed a distinct expression pattern of both cell types (fig. 3a). By comparing the expressed genes (det- $p<0.05) 250$ are exclusively expressed in the UdRPCs and 600 in podocytes (fig. 3b). The most over-represented GO BP-terms exclusive to UdRPCs are associated with cell division and activation of the pre-replicative complex. In comparison the most over-represented GO BP-terms exclusive to podocytes are associated with cell fate commitment, cell morphogenesis and regulation of ion transport (fig. 3c). These genes include important regulators of podocyte morphology and function such as: BCAS3, BDNF, CLIC5, CXCR4, DNM3, GPER1, GRIA3, LCN2, LPAR3, MBL2, NFASC, NRXN1, ROBO1, SH3GL2, SYT1, WNT1, WNT8A, WNT9A, and the collagens COL12A1 and COL14A1. In total, 13762 genes were found to be expressed in common between UdRPCs and podocytes, of these 671 were upregulated and 904 downregulated in podocytes (fig. 3b). The downregulated genes are again associated with cell cycle processes and methylation (fig. 3e). Interestingly genes associated with methylation include DNMT3A, EZH1, MTR, SUZ12, TET2 and genes associated with the cell cycle BUB1, BUB1B, CDC7, CDC25A, FGFR2, MAD2L1, MAP2K6, SIX3, TOP2A, ZBTB16. In contrast, upregulated genes are associated with cell adhesion, positive regulation of cell migration, cell substrate adhesion, morphogenesis of an epithelium and regulation of cytokine production (fig. 3d). These include ARHGEF2, CD44, CXCL16, CX3CL1, DPP4, ETS1, ITGA2, ITGA6, ITGAV, LAMA3, LAMB3, LAMC1, LAMC2, NOTCH1, PDGFA, PDGFB, PODXL, SEMA3A, SEMA3C, WNT7A and VEGFC. Furthermore, genes annotated under the GO BP-term morphogenesis of an epithelium are known to be critical effectors either for epithelial-mesenchymal transition (EMT) or mesenchymal-epithelial transition (MET) ALDH1A3, CAV1, DLG1, FZD7, MICAL2, PDGFA, SMAD3, SMURF1, SMURF2, TGM2, TNFAIP3 and VEGFC. We suggest that our differentiation protocol trigger these processes and thereby mimic a process that is referred to as branching morphogenesis during mammalian kidney development [33]. The generated podocytes express numerous genes which are annotated with neuronal GO BP-terms, such as axonogenesis, axon guidance, chemotaxis, and regulation of axonogenesis (supplementary table 4). We hypothesize that this might reflect the migration process during podocyte maturation or a necessary task during the formation of the foot processes. Additionally, the transcriptomes of UdRPCS UF45, UM48 and UM51, their derived podocytes were compared with expressed genes in iPS cell-derived podocytes, kidney 
biopsy isolated human glomeruli, and mouse podocytes (supplementary fig. 3), as reported by Sharmin et al. [21]. Furthermore, we analysed the expression of several Solute Carrier (SLC) Family members (supplementary fig. 3). Both analyses revealed distinct expression patterns of UdRPCs and their differentiated counterparts. In particular, the SLC genes were found to be exclusively upregulated in podocytes. Key genes associated with the complex podocyte morphology, like PODXL, NPHS1, NPHS2 and SYNPO were found to be upregulated in the differentiated podocytes of all three individuals. Interestingly undifferentiated UdRPCs express CD2AP (an important stabilizer of the slit diaphragm), which seem to be unaffected by our differentiation protocol. In addition, our results provide detailed information about genes exclusively expressed in human UdRPCs which are mesenchymal and differentiated podocyte which are epithelial (supplementary tables 4, 5 and 6).

\section{Effect of Angiotensin II (ANGII) on podocyte morphology and expression of podocyte-specific genes}

Elevated levels of ANGII have been identified as a main risk factor for the initiation and progression of chronic kidney disease (CKD), furthermore increased ANGII concentrations are associated with the downregulation of Nephrin and Synaptopodin expression in podocytes [11, 12]. To evaluate the capacity of our generated podocytes modelling acute and chronic kidney injury, we prepared a final concentration of $100 \mu \mathrm{M}$ ANGII in adv. RPMI medium supplemented with $30 \mu \mathrm{M}$ retinoic acid and treated podocytes derived from three individuals, two-males African (UM48 and UM51) and one female-Caucasian (UF45), for $6 \mathrm{~h}$ and $24 \mathrm{~h}$ respectively. This analysis was performed for podocytes cultured under low (fig. 4) as well as high cell density (supplementary fig. 1). After 6h, dynamic changes in morphology could be observed by immunofluorescence-based detection of $\alpha$-ACTININ expression (fig. 4a + supplementary fig. 1a). While untreated podocytes showed the typical "fried egg" morphology, ANG II treated podocytes derived from all three individuals underwent massive disruption of the cytoskeleton, resulting in the inhibition of podocyte spreading and subsequent loss of foot processes as observed by the round and condensed morphology (fig. 4a supplementary fig. 1a). To confirm that the disruptive effect is indeed mediated by ANGII, the effects on the cytoskeleton were evaluated by gene-specific mRNA expression of both ANGII receptors, AGTR1 and AGTR2, as well as podocyte key structural proteins NPHS1 and SNYPO and the pro-inflammatory cytokine IL-6 (fig. 4b-f). While AGTR1 expression was found not to be significantly downregulated, AGTR2 was found to be upregulated in podocytes derived under low density cultivation from all three individuals after $6 \mathrm{~h}$ (fig. $4 \mathrm{~b}-\mathrm{c}$ ). Interestingly when the cells were cultured under high density conditions both receptors seemed to be upregulated due to the ANGII treatment after $6 \mathrm{~h}$, with the only exception for AGTR2 in UF45 podocytes (supplementary fig. 1b-c). When the ANGII treatment was prolonged to $24 \mathrm{~h}$, the mRNA expression level of AGTR1 was still downregulated in UM48 podocytes cultured under low density conditions, at a similar level as in $6 \mathrm{~h}$, while UM51 podocytes 
show a modest increase 0.6 -fold. mRNA expression of NPHS1 and SYNPO was downregulated upon ANGII treatment in all individuals and culture conditions, except for the SYNPO expression in podocytes UF45 under high density cultivation which was upregulated (fig. 4 d-e and supplementary fig. 1d-e). Podocytes cultured under low density conditions showed only minor changes in the NPHS1 and SYNPO expression after $6 \mathrm{~h}$ of ANGII treatment, while mRNA expression in UM51 and UM48 podocytes cultured under high density was found to be downregulated by 2.6- and 1.7-fold (NPHS1) and 2.6- and 1-fold (SYNPO) respectively (supplementary fig. 1b-c). When ANGII treatment was prolonged to $24 \mathrm{~h}$ under low density conditions, UM48 and UM51 podocytes both showed downregulated expression of NPHS1 expression, 0.9 -fold, and 3-fold, respectively. In contrast expression of SYNPO remained at similar levels seen in $6 \mathrm{~h}$ of ANGII treatment. Since ANGII is a proinflammatory factor we also evaluated mRNA expression of IL-6 in UF45, UM48 and UM51 podocytes after $6 \mathrm{~h}$ and UM48 and UM51 after $24 \mathrm{~h}$ of ANGII treatment (fig. 4f). IL-6 mRNA levels were found not to be significantly regulated after 6h (UF45: 0.9-fold; UM48: 0.3-fold and UM51: 0.3-fold) or 24h of ANGII treatment (UM48: 0.3-fold and UM51: 0.5 -fold). To evaluate whether the transcriptional changes of cytoskeleton-associated genes could be in part mediated by epigenetic modifications, we applied bisulfite genomic sequencing to analyse the methylation status of a CpG-rich region of the NPHS2 promoter. In total a $415 \mathrm{bp}$ long NPHS2 fragment $11 \mathrm{bp}$ upstream of the TSS, spanning $23 \mathrm{CpG}$ dinucleotides, was analyzed and this revealed a lack of methylation changes occurring during $6 \mathrm{~h}$ of ANGII treatment in UM51 podocyte (supplementary fig. 2).

\section{Effects of Angiotensin II on the transcriptome and secretome of urine derived podocytes}

To further investigate the effect of ANGII, we performed a comparative transcriptome analysis of UM48 and UM51 podocytes after $6 \mathrm{~h}$ and $24 \mathrm{~h}$ of ANGII treatment ( $\mathrm{n}=2$ biological replicates per genotype). Hierarchical clustering analysis comparing the transcriptomes of non-treated and treated podocytes revealed distinct transcriptomes for the three conditions, without treatment, $6 \mathrm{~h}$ and $24 \mathrm{~h}$ of ANGII treatment respectively (fig. 5a). Interestingly while podocytes treated for $6 \mathrm{~h}$ seem to cluster rather by their genetic background, the cells treated for $24 \mathrm{~h}$ formed a distinct cluster. Further analyses identified 478 genes (det- $p<0.05$ ) exclusively expressed in the control conditions and 63 genes in the $6 \mathrm{~h}$ Angiotensin conditions (fig. 5b). The most over-represented GO BP-terms derived from the 478 genes are associated with growth and locomotion (fig. $5 \mathrm{~d}$ ). These genes include BDNF, DNAH5, TTC21A and VPS13A. In contrast the most over-represented GO BP-terms linked to the 63 are associated with immune system process, response to stimulus and cell proliferation (fig. $5 \mathrm{~d}$ ). These genes include GATA2, GLI3, IL12RB1, LILRB2, PECAM-1, SIX3 and VTCN1. In total 13783 nonregulated genes were found to be expressed in common between both conditions. By comparing the expressed genes (det-p < 0.05) after $24 \mathrm{~h}$ of ANGII treatment, 339 genes were found to be exclusively expressed in the control podocytes and 335 genes in the treated cells (fig. 5c). The most over- 
represented GO BP-terms of the 339 genes are associated with multi-organism and multicellular organismal process and locomotion (fig. 5e). These genes include ASPM, CAMK2B, HTR6, ITPR1, ISL1, ISL2, KCNMB4, NCAPH, ROBO1, SCN3B, SHANK3, SLIT1, TOPA2 and TYRO3. The full gene list can be found in supplementary table 7. In total 13922 genes were found to be expressed in common between both conditions, from which 1287 were upregulated and 1100 downregulated after $24 \mathrm{~h}$ of ANGII treatment (supplementary tables $8+9$ ). The upregulated genes are associated with cell cycle, DNA repair, cell cycle phase transition, methylation, and regulation of cellular response to stress (fig. 5f). These include ALMS1, ATF4, CDK6, DDIT3, EDN1, H2AC4, H2BC14, H3C7, FANCD2, FOXM1, MSH2, MSH6, NUP35, NUP37, NUP54, NUP107, NUP133, NUP153, NUP155, NUP214, PARP2, PCNA, PLK2, PRC1, RAD21, RAD51C, SNAI1, SNAI2, SMC2, SMC4, SUZ12, TGFB2, WDR70, WNT5A and XRCC4. Interestingly genes annotated for the GO BP-terms cell cycle and methylation were also found in the undifferentiated UdRPCs. The downregulated genes after 24h ANGII treatment are associated with regulation of cell adhesion, cell junction organization, axonogenesis, actin cytoskeleton organization, tissue-, and gland morphogenesis (fig. $5 \mathrm{~g}$ ). Genes annotated under the GO BP-term regulation of cell adhesion include AFDN, CD74, CORO1A, CORO2B, DDR1, DPP4, EPCAM, ITGA2, JAG1, KLF4, LAMA3, LAMA5, LDLR, NLRP3 and PODXL. Genes annotated under the GO BP-term cell junction organization include COL17A1, CLDN3, LAMB3 and LAMC2. Genes annotated under the GO BP-term tissue and gland morphogenesis include CXCL16, ELF3, NTN4, PAX6, PLAU, SEMA3A, SLC9A3R1, SMURF1, TUBB2B, VANGL2, WNT7A, WNT7B and WNT10A. Of note, expression of CXCL16, DPP4, ITAG2, LAMA3, LAM3B, PODXL, ROBO1, SEMA3A, SMURF1 and WNT7A were found to be upregulated upon differentiation of UdRPCs into podocytes, but then downregulated upon ANGII treatment. This finding may suggest that these genes might have major implications during the differentiation of UdRPCs into podocytes and ANGII induced podocyte injury. Finally, expression of numerous genes annotated under the GO BP-term actin cytoskeleton organization were downregulated upon $24 \mathrm{~h}$ ANGII treatment and revealed a distinct clustering of UdRPCS, Podocytes and Podocytes treated with ANGII for $24 \mathrm{~h}$. The full gene list is given in supplementary tables $4+9$. Interesting to note most of these genes also show basal levels of expression in undifferentiated UdRPCs. Genes annotated for the GO BP-term actin cytoskeleton organization include ANKRD13D, ANKRD27, COL5A2, KRT17, KRT19, NRCAM, SEMA3A, SEMA3F, SEMA4C, SEMA5A, SEMA6D, SEMA7A, SLC9A3R1, SLC12A7, SLC12A8, TICAM1 and VANGL2. These findings further emphasize the disruptive effect of ANGII on cytoskeleton of podocytes as it has been observed by immunofluorescence-based detection of protein expression and q-RT PCR. An overview of the KEGG annotated renin-angiotensin system is given in figure $4 \mathrm{~g}$. 
By analysing the secretomes of UM48 and UM51 podocytes with and without $6 \mathrm{~h}$ and $24 \mathrm{~h}$ of ANGII treatment, a plethora of significantly regulated secreted factors, specific for human kidney and associated with renal diseases, were identified (supplementary fig. 4). In accord with the transcriptome data only minor changes after $6 \mathrm{~h}$ of ANGII treatment could be observed with only three cytokines significantly altered, ADIPOQ, ANPEP and AGER. Major alterations within the secretome were manifested after $24 \mathrm{~h}$, resulting in a significant up-regulation of ANPEP, CCN1, IL6, MMP9, SERPINA3 and VEGF and down-regulation of CXCL16, REN, TNF $\alpha$, TFF3 and PLAU.

\section{Discussion}

The kidney glomerulus or renal corpuscle consists of a glomerular tuft and the Bowman's capsule. Its major task is the filtration of blood to generate urine and it consists of four distinct cell types endothelial cells, mesangial cells, parietal epithelial cells of Bowman's capsule and podocytes. In the present manuscript, we present a differentiation protocol for the successful and reproducible differentiation of UdRPCs into podocytes. These podocytes have the typical complex architecture and express podocyte specific proteins Synaptopodin, Nephrin and Podocin, which are necessary for the establishment of either the slit diaphragm or the foot processes. Podocytes are major contributors towards balancing colloidal pressure within the renal system and transport metabolites by endo/exocytosis of Albumin. As means of diagnosis, the quantitative analysis of Albumin in urine is used as a mark of renal cell functionality. The Albumin infiltration pathway partly takes place within podocytes in vivo and in vitro [34-36]. The comparison of the transcriptomes pertaining to UdRPC differentiated podocytes with previously reported data-sets of iPSC-derived podocytes, biopsy derived human glomeruli, and mouse podocytes [21], revealed distinct clustering of UdRPCs and their differentiated counterparts, whereas most of the genes were found to be upregulated after our differentiation protocol. Furthermore, genes that are exclusively expressed or upregulated (det$p<0.05)$ after our differentiation protocol, but are not on the reported list [21], have been shown to be critical for podocyte morphology and/or function. Unique genes from our data that are associated with the filtration barrier or cytoskeleton of podocytes include the genes CX3CL1 [37], DNM3 [38], DPP4 [39] and NRX1 [40]. Unique genes from our data that are associated with podocyte function or survival include BDNF [41], CXCL16, [42] GRIA3 [43], PDGF-C [44], RAB3A [45] and VEGF-C [46]. Members of Semaphorin gene family SEMA3A and SEMA3C were also significantly upregulated. Both are guidance proteins, expressed during kidney development and regulate kidney vascular patterning, endothelial cell migration, survival, uteric bud branching and podocyte-endothelial crosstalk [47]. In a mouse model of semaphorin3a gain- and loss of function experiments revealed a dose dependent role of Semaphorin3a in podocyte differentiation and establishment of the glomerular filtration barrier [48]. 
During renal development the glomerulus is established in four stages: the renal vesicle stage, the Sshaped body stage, the capillary loop stage, and the maturing-glomeruli stage [33]. This developmental process is initiated by the metanephric mesenchyme. It induces the ureteric bud epithelium to grow and finally to branch and form the collecting duct system of the kidney. Hereby the mesenchymal cells of the metanephric mesenchyme have to form the polarized epithelial cells of the renal vesicle and therefore undergo a process that is referred to as mesenchymal-to-epithelial transition (MET) [49]. The podocytes in the glomerulus are generated by a population of cells within the pariental epithelium. These cells are thought to migrate into the glomerular tuft and then differentiate into the mesenchyme like podocytes $[50,51]$. Therefore, the maturation of podocytes is associated with a physiological epithelial-to-mesenchymal transition [1]. These developmental processes are also reflected in our transcriptome data. Genes that are upregulated in the derived podocytes are annotated with the GO BP-terms such as axon guidance and chemotaxis, thus enabling speculation that this might reflect the migration process during podocyte maturation. In addition, as differentiation into podocytes also involves both MET and EMT, it is not surprising that the upregulated data set consists of numerous critical effectors for both processes, for instance wnt-1 has been reported to be a major driver of MET [52]. Interestingly the genes Cav1 and Dlg1 have been linked to kidney development and renal pathological conditions in mice $[49,53]$. Further signs of EMT/MET are the differential expression of cell-cell contact-associated markers and functional changes associated with the conversion between mobile and stationary cells [54]. Confirmation that these changes are induced by our differentiation protocol is reflected in the podocyte upregulated dataset by the annotated GO BP-terms cell adhesion, cell substrate adhesion, as well as the podocyte exclusive expression of the collagens COL12A1 and COL14A1.

Developmental processes are regulated by epigenetic mechanisms and these are of fundamental importance for cellular differentiation [55]. Epigenetic remodeling is associated with the establishment and removal of histone modifications and DNA-methylation, to generate the cell typespecific epigenome. The epigenetic remodeling needed for the differentiation of UdRPCs into podocytes is also reflected by our transcriptome data. We observed downregulation of the de novo DNA-methyltransferase 3a (DNMT3a) as well as Tet Methylcytosine Dioxygenase 2 (TET2) in podocytes. Whilst DNMT3a is required for the establishment of CpG methylation during embryogenesis [56], the members of the TET family are needed for the initiation of demethylation $[57,58]$. This targeted epigenetic remodeling during the differentiation process is manifested at the WT1 promoter, as observed by bisulfite sequencing, therefore lending support to the speculation that this process is mediated by TET2. Furthermore, genes associated with to the polycomb repressive complex, EZH1 and SUZ12 [59], were also found to be downregulated. Specifically, EZH1 has been reported to be important for the maintenance of stem cell identity and execution of 
pluripotency [60]. Podocytes are terminally differentiated cell, which are unable to undergo cell division in vivo [1]. Due to the observed downregulation of genes associated with epigenetic modifications and cell cycle processes, we propose that the podocytes generated with our protocol have acquired this terminally differentiated state. This hypothesis is further strengthened by the observed downregulation of MSC associated cell cycle related genes BUBR1, CDC7, CDC25A, MAD2L1, FGFR2, SIX3 and TOPA2. Furthermore, these genes have been reported to maintain stemness in mesenchymal stem cells and their downregulation is linked to the differentiation of MSCs [61-68].

Of note the derived podocytes were found to have upregulated the mRNA expression of several solute carrier family members (SLC) (supplementary fig. 3). To our knowledge this is the first report describing the expression of SLCS in human urine-derived podocytes. We propose that the abundance of solute carrier family members is due to the increase in transmembrane transport, as indicated by our podocyte exclusive GO BP-terms, regulation of ion transmembrane transport, neurotransmitter transport, organic acid transport and chemical synaptic transmission. Furthermore, SLCs have been shown to be involved in human diseases and drug targets, thus establishing our human urine-derived podocytes as a valuable cellular tool for studying SLCs in renal development and under pathological conditions [69].

The renin-angiotensin system (RAS) major implications in humans is the maintenance of plasma sodium concentration, arterial blood pressure and extracellular volume. Activation of the RAS leads to hypertension, cell proliferation, inflammation, and fibrosis and therefore has implications for all tissues of the body [70]. Angiotensin receptors and especially AGTR1 in the kidney have been reported to be primarily causative for hypertension in mammals [71] and are associated with renal sodium handling. AGTRs therefore have been recognized to be commonly expressed in several segments of the nephron including the thick ascending limb, distal tubule, collecting duct and renal vasculature [72-77]. The stimulation of these receptors has been linked to renal vasoconstriction, reduced medullary blood flow, diminishing the renal capacity of sodium handling [78-80], leading to podocyte injury and loss [15]. The main mediator of RAS is ANGII and it has been shown that ANG receptors within the kidney are the main mediators of hypertension [71]. Elevated levels of circulating and intracellular ANGII and aldosterone lead to pro-fibrotic, -inflammatory and hypertrophic milieu that causes remodelling and dysfunction in cardiovascular and renal tissues [81]. The major finding in this study is the disruptive effect of ANGII on the cytoskeleton of podocyte. Since ANGII obviously interferes with the podocyte cytoskeleton, it is important to note that commonly housekeeping genes like $\beta$-ACTIN are unsuitable for normalization and we therefore used $R P L O$, which should not be affected. Employing immunofluorescence-based protein expression detection we observed cytoskeletal changes of the normal morphology of podocytes, leading to the 
inhibition of podocyte spreading and down-regulated expression of NPHS1 and SYNPO. The downregulated expression of NPHS1 and SYNPO has been reported as a hallmark of podocyte injury [13] and is causative for the disruption of foot processes (FPs) and the slit diaphragm (SD). This effect also probably due to the downregulated expression of numerous genes associated with the GO-BP-actin cytoskeleton organization (fig. $5 \mathrm{~g}$ and supplementary table 9). As an example instance Alpha-Actinin4 (ACTN4) is highly expressed in podocyte FPs and NPHS1 is connected to the actin cytoskeleton thus contributing to podocyte actin dynamics, signaling and mobility $[2,3,6]$. Furthermore, our transcriptome data unveiled the downregulated expression of CORO2B, DPP4, ELF3, LDLR, KLF4, ROBO1 and WNT7A. These genes have been linked to podocyte injury and filtration barrier impairments [18, 39, 82-86]. Of note, KLF4 has been shown to directly regulate NPHS1 expression [39], thus suggesting that the disruptive effect of ANGII might be amongst others also mediated by KLF4. In the upregulated gene set numerous genes associated with cell cycle initiation and DNA double strand repair, such as FANCD2, MSH2, WDR7 and XRCC4 (fig. $5 \mathrm{f}$ and supplementary table 8). In a mouse model of Brand et al., it was shown that ANGII is indeed able to induce DNA damage via a dose dependent increase of oxidative stress, which results in DNA damage [87]. Furthermore, elevated levels of ATF4, EDN-1, TGFB2 and PLK2 have all been linked to podocyte injury and loss of key proteins necessary for the maintenance of the complex podocyte architecture [88-91]. Most of the cytoskeleton-related genes that were downregulated upon ANGII treatment in podocytes, had lower levels of expression in the undifferentiated UdRPCs. Although the expression of Ankyrins, Collagens, Keratins and Semaphorins have been linked to kidney development and functionality [38, $47,48,92]$, to our knowledge only Semaphorins have been reported to be involved in kidney injury $[47,93,94]$. Of note numerous members of the sodium and chloride symporter family SLC6 [69], are highly upregulated in the differentiated podocytes (supplementary fig. 3), while upon ANGII stimulation upregulated expression of AGTR1 and 2 was observed. We conclude that our findings offer the possibility of establishing biomarkers indicative of differentiation of UdRPCs into podocytes as well as initiation and progression of CKD.

To surmise, we have described a differentiation protocol for the successful and reproducible differentiation of SIX2-positive urine derived renal progenitor cells (UdRPCs) from two African males aged 48 and 51 and one female Caucasian aged 45 years into mature podocytes with the ability to execute Albumin endocytosis. UdRPCs enable the unique possibility to study nephrogenesis and associated diseases thus obviating the need of iPSCs. Furthermore, the responsiveness of UdRPC derived podocytes to ANGII implies potential applications in nephrotoxicity studies and drug screening. Figure 6 presents a graphical summary of this study. 


\section{Acknowledgements}

James Adjaye acknowledges funding from the medical faculty of Heinrich Heine UniversityDuesseldorf, Germany

\section{Supporting information}

Supporting information in the supplementary section or from the author.

\section{Conflict of interest}

The authors declare no conflict of interest.

\section{References}

1. Greka A, Mundel P. Cell biology and pathology of podocytes. Annu Rev Physiol. 2012;74:299323. doi:10.1146/annurev-physiol-020911-153238.

2. Critchley DR. Focal adhesions - the cytoskeletal connection. Current Opinion in Cell Biology. 2000;12:133-9. doi:10.1016/S0955-0674(99)00067-8.

3. El-Husseini AE, Kwasnicka D, Yamada T, Hirohashi $S$, Vincent SR. BERP, a novel ring finger protein, binds to alpha-actinin-4. Biochem Biophys Res Commun. 2000;267:906-11. doi:10.1006/bbrc.1999.2045.

4. Kestilä M, Lenkkeri U, Männikkö M, Lamerdin J, McCready P, Putaala H, et al. Positionally Cloned Gene for a Novel Glomerular Protein-Nephrin-Is Mutated in Congenital Nephrotic Syndrome. Molecular Cell. 1998;1:575-82. doi:10.1016/S1097-2765(00)80057-X.

5. Boute N, Gribouval O, Roselli S, Benessy F, Lee H, Fuchshuber A, et al. NPHS2, encoding the glomerular protein podocin, is mutated in autosomal recessive steroid-resistant nephrotic syndrome. Nat Genet. 2000;24:349-54. doi:10.1038/74166.

6. Tryggvason K, Pikkarainen T, Patrakka J. Nck links nephrin to actin in kidney podocytes. Cell. 2006;125:221-4. doi:10.1016/j.cell.2006.04.002.

7. Carey RM, Whelton PK. Prevention, Detection, Evaluation, and Management of High Blood Pressure in Adults: Synopsis of the 2017 American College of Cardiology/American Heart Association Hypertension Guideline. Ann Intern Med. 2018;168:351-8. doi:10.7326/M17-3203.

8. Ito M, Oliverio MI, Mannon PJ, Best CF, Maeda N, Smithies O, Coffman TM. Regulation of blood pressure by the type 1A angiotensin II receptor gene. Proc Natl Acad Sci U S A. 1995;92:3521-5. doi:10.1073/pnas.92.8.3521.

9. Shanmugam S, Sandberg K. Ontogeny of angiotensin II receptors. Cell Biol Int. 1996;20:169-76. doi:10.1006/cbir.1996.0021.

10. Sharma M, Sharma R, Greene AS, McCarthy ET, Savin VJ. Documentation of angiotensin II receptors in glomerular epithelial cells. Am J Physiol. 1998;274:F623-7. doi:10.1152/ajprenal.1998.274.3.f623.

11. Jia J, Ding G, Zhu J, Chen C, Liang W, Franki N, Singhal PC. Angiotensin II infusion induces nephrin expression changes and podocyte apoptosis. Am J Nephrol. 2008;28:500-7. doi:10.1159/000113538.

12. Liu X, Zhang $H$, Wang $Q$, Yu K, Wang R, Sun J. Blockade of vascular endothelial growth factorA/receptor 2 exhibits a protective effect on angiotensin-II stimulated podocytes. Mol Med Rep. 2015;12:4340-5. doi:10.3892/mmr.2015.3911.

13. Rüster $C$, Wolf $G$. Renin-angiotensin-aldosterone system and progression of renal disease. J Am Soc Nephrol. 2006;17:2985-91. doi:10.1681/ASN.2006040356. 
14. Ding G, Reddy K, Kapasi AA, Franki N, Gibbons N, Kasinath BS, Singhal PC. Angiotensin II induces apoptosis in rat glomerular epithelial cells. Am J Physiol Renal Physiol. 2002;283:F173-80. doi:10.1152/ajprenal.00240.2001.

15. Kriz W, Gretz N, Lemley KV. Progression of glomerular diseases: is the podocyte the culprit? Kidney Int. 1998;54:687-97. doi:10.1046/j.1523-1755.1998.00044.x.

16. Saleem MA, O'Hare MJ, Reiser J, Coward RJ, Inward CD, Farren T, et al. A conditionally immortalized human podocyte cell line demonstrating nephrin and podocin expression. J Am Soc Nephrol. 2002;13:630-8.

17. Srivastava T, Sharma M, Yew K-H, Sharma R, Duncan RS, Saleem MA, et al. LPS and PAN-induced podocyte injury in an in vitro model of minimal change disease: changes in TLR profile. J Cell Commun Signal. 2013;7:49-60. doi:10.1007/s12079-012-0184-0.

18. Yang Y, Yang Q, Yang J, Ma Y, Ding G. Angiotensin II induces cholesterol accumulation and injury in podocytes. Sci Rep. 2017;7:10672. doi:10.1038/s41598-017-09733-w.

19. Musah S, Dimitrakakis N, Camacho DM, Church GM, Ingber DE. Directed differentiation of human induced pluripotent stem cells into mature kidney podocytes and establishment of a Glomerulus Chip. Nat Protoc. 2018;13:1662-85. doi:10.1038/s41596-018-0007-8.

20. Freedman BS, Brooks CR, Lam AQ, Fu H, Morizane R, Agrawal V, et al. Modelling kidney disease with CRISPR-mutant kidney organoids derived from human pluripotent epiblast spheroids. Nat Commun. 2015;6:8715. doi:10.1038/ncomms9715.

21. Sharmin S, Taguchi A, Kaku Y, Yoshimura Y, Ohmori T, Sakuma T, et al. Human Induced Pluripotent Stem Cell-Derived Podocytes Mature into Vascularized Glomeruli upon Experimental Transplantation. J Am Soc Nephrol. 2016;27:1778-91. doi:10.1681/ASN.2015010096.

22. Taguchi A, Kaku Y, Ohmori T, Sharmin S, Ogawa M, Sasaki H, Nishinakamura R. Redefining the in vivo origin of metanephric nephron progenitors enables generation of complex kidney structures from pluripotent stem cells. Cell Stem Cell. 2014;14:53-67. doi:10.1016/j.stem.2013.11.010.

23. Takasato M, Er PX, Chiu HS, Maier B, Baillie GJ, Ferguson C, et al. Kidney organoids from human iPS cells contain multiple lineages and model human nephrogenesis. Nature. 2015;526:564-8. doi:10.1038/nature15695.

24. Rahman MS, Wruck W, Spitzhorn L-S, Nguyen L, Bohndorf M, Martins S, et al. The FGF, TGF $\beta$ and WNT axis Modulate Self-renewal of Human SIX2+ Urine Derived Renal Progenitor Cells. Sci Rep. 2020;10:739. doi:10.1038/s41598-020-57723-2.

25. Santourlidis S, Trompeter H-I, Weinhold S, Eisermann B, Meyer KL, Wernet P, Uhrberg M. Crucial role of DNA methylation in determination of clonally distributed killer cell Ig-like receptor expression patterns in NK cells. J Immunol. 2002;169:4253-61. doi:10.4049/jimmunol.169.8.4253.

26. Zhou Y, Zhou B, Pache L, Chang M, Khodabakhshi AH, Tanaseichuk O, et al. Metascape provides a biologist-oriented resource for the analysis of systems-level datasets. Nat Commun. 2019;10:1523. doi:10.1038/s41467-019-09234-6.

27. Schneider CA, Rasband WS, Eliceiri KW. NIH Image to ImageJ: 25 years of image analysis. Nat Methods. 2012;9:671-5. doi:10.1038/nmeth.2089.

28. Gentleman RC, Carey VJ, Bates DM, Bolstad B, Dettling M, Dudoit S, et al. Bioconductor: open software development for computational biology and bioinformatics. Genome Biol. 2004;5:R80. doi:10.1186/gb-2004-5-10-r80.

29. Du $P$, Kibbe WA, Lin SM. lumi: a pipeline for processing Illumina microarray. Bioinformatics. 2008;24:1547-8. doi:10.1093/bioinformatics/btn224.

30. Graffmann N, Ring S, Kawala M-A, Wruck W, Ncube A, Trompeter H-I, Adjaye J. Modeling Nonalcoholic Fatty Liver Disease with Human Pluripotent Stem Cell-Derived Immature Hepatocyte-Like Cells Reveals Activation of PLIN2 and Confirms Regulatory Functions of 
Peroxisome Proliferator-Activated Receptor Alpha. Stem Cells Dev. 2016;25:1119-33. doi:10.1089/scd.2015.0383.

31. Kanehisa M, Furumichi M, Tanabe M, Sato $Y$, Morishima K. KEGG: new perspectives on genomes, pathways, diseases and drugs. Nucleic Acids Res. 2017;45:D353-D361. doi:10.1093/nar/gkw1092.

32. Carvalho BS, Irizarry RA. A framework for oligonucleotide microarray preprocessing. Bioinformatics. 2010;26:2363-7. doi:10.1093/bioinformatics/btq431.

33. Saxén L, Sariola H. Early organogenesis of the kidney. Pediatr Nephrol. 1987;1:385-92. doi:10.1007/BF00849241.

34. Birn H, Christensen El. Renal albumin absorption in physiology and pathology. Kidney Int. 2006;69:440-9. doi:10.1038/sj.ki.5000141.

35. Eyre J, loannou K, Grubb BD, Saleem MA, Mathieson PW, Brunskill NJ, et al. Statin-sensitive endocytosis of albumin by glomerular podocytes. Am J Physiol Renal Physiol. 2007;292:F674-81. doi:10.1152/ajprenal.00272.2006.

36. Tojo A, Kinugasa S. Mechanisms of glomerular albumin filtration and tubular reabsorption. Int J Nephrol. 2012;2012:481520. doi:10.1155/2012/481520.

37. Katsuyama K, Fujinaka H, Yamamoto K, Nameta M, Yaoita E, Yoshida $Y$, et al. Expression of the chemokine fractalkine (FKN/CX3CL1) by podocytes in normal and proteinuric rat kidney glomerulus. Nephron Exp Nephrol. 2009;113:e45-56. doi:10.1159/000228408.

38. Miao J, Fan Q, Cui Q, Zhang H, Chen L, Wang S, et al. Newly identified cytoskeletal components are associated with dynamic changes of podocyte foot processes. Nephrol Dial Transplant. 2009;24:3297-305. doi:10.1093/ndt/gfp338.

39. Nistala R, Habibi J, Aroor A, Sowers JR, Hayden MR, Meuth A, et al. DPP4 inhibition attenuates filtration barrier injury and oxidant stress in the zucker obese rat. Obesity (Silver Spring). 2014;22:2172-9. doi:10.1002/oby.20833.

40. Saito A, Miyauchi N, Hashimoto T, Karasawa T, Han GD, Kayaba M, et al. Neurexin-1, a presynaptic adhesion molecule, localizes at the slit diaphragm of the glomerular podocytes in kidneys. Am J Physiol Regul Integr Comp Physiol. 2011;300:R340-8. doi:10.1152/ajpregu.00640.2009.

41. Endlich N, Lange T, Kuhn J, Klemm P, Kotb AM, Siegerist F, et al. BDNF: mRNA expression in urine cells of patients with chronic kidney disease and its role in kidney function. J Cell Mol Med. 2018;22:5265-77. doi:10.1111/jcmm.13762.

42. Gutwein P, Abdel-Bakky MS, Schramme A, Doberstein K, Kämpfer-Kolb N, Amann K, et al. CXCL16 is expressed in podocytes and acts as a scavenger receptor for oxidized low-density lipoprotein. Am J Pathol. 2009;174:2061-72. doi:10.2353/ajpath.2009.080960.

43. Li Z, Chen H, Zhong F, Zhang W, Lee K, He JC. Expression of Glutamate Receptor Subtype 3 Is Epigenetically Regulated in Podocytes under Diabetic Conditions. Kidney Dis (Basel). 2019;5:3442. doi:10.1159/000492933.

44. Eitner F, Ostendorf T, Kretzler M, Cohen CD, Eriksson U, Gröne H-J, Floege J. PDGF-C expression in the developing and normal adult human kidney and in glomerular diseases. J Am Soc Nephrol. 2003;14:1145-53. doi:10.1097/01.asn.0000062964.75006.a8.

45. Rastaldi MP, Armelloni S, Berra S, Calvaresi N, Corbelli A, Giardino LA, et al. Glomerular podocytes contain neuron-like functional synaptic vesicles. FASEB J. 2006;20:976-8. doi:10.1096/fj.05-4962fje.

46. Müller-Deile J, Worthmann K, Saleem M, Tossidou I, Haller H, Schiffer M. The balance of autocrine VEGF-A and VEGF-C determines podocyte survival. Am J Physiol Renal Physiol. 2009;297:F1656-67. doi:10.1152/ajprenal.00275.2009. 
47. Reidy K, Tufro A. Semaphorins in kidney development and disease: modulators of ureteric bud branching, vascular morphogenesis, and podocyte-endothelial crosstalk. Pediatr Nephrol. 2011;26:1407-12. doi:10.1007/s00467-011-1769-1.

48. Reidy KJ, Villegas G, Teichman J, Veron D, Shen W, Jimenez J, et al. Semaphorin3a regulates endothelial cell number and podocyte differentiation during glomerular development. Development. 2009;136:3979-89. doi:10.1242/dev.037267.

49. Naim E, Bernstein A, Bertram JF, Caruana G. Mutagenesis of the epithelial polarity gene, discs large 1, perturbs nephrogenesis in the developing mouse kidney. Kidney Int. 2005;68:955-65. doi:10.1111/j.1523-1755.2005.00489.x.

50. Smeets B, Uhlig S, Fuss A, Mooren F, Wetzels JFM, Floege J, Moeller MJ. Tracing the origin of glomerular extracapillary lesions from parietal epithelial cells. J Am Soc Nephrol. 2009;20:260415. doi:10.1681/ASN.2009010122.

51. Appel D, Kershaw DB, Smeets B, Yuan G, Fuss A, Frye B, et al. Recruitment of podocytes from glomerular parietal epithelial cells. J Am Soc Nephrol. 2009;20:333-43. doi:10.1681/ASN.2008070795.

52. Hay ED, Zuk A. Transformations between epithelium and mesenchyme: Normal, pathological, and experimentally induced. American Journal of Kidney Diseases. 1995;26:678-90. doi:10.1016/0272-6386(95)90610-X.

53. Strippoli R, Loureiro J, Moreno V, Benedicto I, Pérez Lozano ML, Barreiro O, et al. Caveolin-1 deficiency induces a MEK-ERK1/2-Snail-1-dependent epithelial-mesenchymal transition and fibrosis during peritoneal dialysis. EMBO Mol Med. 2015;7:102-23. doi:10.15252/emmm.201404127.

54. Yang J, Weinberg RA. Epithelial-mesenchymal transition: at the crossroads of development and tumor metastasis. Dev Cell. 2008;14:818-29. doi:10.1016/j.devcel.2008.05.009.

55. Berdasco M, Esteller M. Aberrant epigenetic landscape in cancer: how cellular identity goes awry. Dev Cell. 2010;19:698-711. doi:10.1016/j.devcel.2010.10.005.

56. Jair K-W, Bachman KE, Suzuki H, Ting AH, Rhee I, Yen R-WC, et al. De novo CpG island methylation in human cancer cells. Cancer Res. 2006;66:682-92. doi:10.1158/0008-5472.CAN05-1980.

57. Tahiliani M, Koh KP, Shen Y, Pastor WA, Bandukwala H, Brudno Y, et al. Conversion of 5methylcytosine to 5-hydroxymethylcytosine in mammalian DNA by MLL partner TET1. Science. 2009;324:930-5. doi:10.1126/science.1170116.

58. Ito S, D'Alessio AC, Taranova OV, Hong K, Sowers LC, Zhang Y. Role of Tet proteins in $5 \mathrm{mC}$ to $5 \mathrm{hmC}$ conversion, ES-cell self-renewal and inner cell mass specification. Nature. 2010;466:112933. doi:10.1038/nature09303.

59. Klutstein M, Nejman D, Greenfield R, Cedar H. DNA Methylation in Cancer and Aging. Cancer Res. 2016;76:3446-50. doi:10.1158/0008-5472.CAN-15-3278.

60. Shen X, Liu Y, Hsu Y-J, Fujiwara Y, Kim J, Mao X, et al. EZH1 mediates methylation on histone H3 lysine 27 and complements EZH2 in maintaining stem cell identity and executing pluripotency. Molecular Cell. 2008;32:491-502. doi:10.1016/j.molcel.2008.10.016.

61. Lee J, Lee CG, Lee K-W, Lee C-W. Cross-talk between BubR1 expression and the commitment to differentiate in adipose-derived mesenchymal stem cells. Exp Mol Med. 2009;41:873-9. doi:10.3858/emm.2009.41.12.093.

62. Qiu W, Kassem M. miR-141-3p inhibits human stromal (mesenchymal) stem cell proliferation and differentiation. Biochim Biophys Acta. 2014;1843:2114-21. doi:10.1016/j.bbamcr.2014.06.004.

63. Guan X, Gao Y, Zhou J, Wang J, Zheng F, Guo F, et al. miR-223 Regulates Adipogenic and Osteogenic Differentiation of Mesenchymal Stem Cells Through a C/EBPs/miR-223/FGFR2 Regulatory Feedback Loop. Stem Cells. 2015;33:1589-600. doi:10.1002/stem.1947. 
64. Lu G-M, Rong Y-X, Liang Z-J, Hunag D-L, Ma Y-F, Luo Z-Z, et al. Multiomics global landscape of stemness-related gene clusters in adipose-derived mesenchymal stem cells. Stem Cell Res Ther. 2020;11:310. doi:10.1186/s13287-020-01823-3.

65. Diacou R, Zhao Y, Zheng D, Cvekl A, Liu W. Six3 and Six6 Are Jointly Required for the Maintenance of Multipotent Retinal Progenitors through Both Positive and Negative Regulation. Cell Rep. 2018;25:2510-2523.e4. doi:10.1016/j.celrep.2018.10.106.

66. Shi N, Xie W-B, Chen S-Y. Cell division cycle 7 is a novel regulator of transforming growth factor$\beta$-induced smooth muscle cell differentiation. J Biol Chem. 2012;287:6860-7. doi:10.1074/jbc.M111.306209.

67. Ullah M, Stich S, Notter M, Eucker J, Sittinger M, Ringe J. Transdifferentiation of mesenchymal stem cells-derived adipogenic-differentiated cells into osteogenic- or chondrogenicdifferentiated cells proceeds via dedifferentiation and have a correlation with cell cycle arresting and driving genes. Differentiation. 2013;85:78-90. doi:10.1016/j.diff.2013.02.001.

68. Zaim M, Isik S. DNA topoisomerase $\| \beta$ stimulates neurite outgrowth in neural differentiated human mesenchymal stem cells through regulation of Rho-GTPases (RhoA/Rock2 pathway) and Nurr1 expression. Stem Cell Res Ther. 2018;9:114. doi:10.1186/s13287-018-0859-4.

69. César-Razquin A, Snijder B, Frappier-Brinton T, Isserlin R, Gyimesi G, Bai X, et al. A Call for Systematic Research on Solute Carriers. Cell. 2015;162:478-87. doi:10.1016/j.cell.2015.07.022.

70. Patel S, Rauf A, Khan H, Abu-lzneid T. Renin-angiotensin-aldosterone (RAAS): The ubiquitous system for homeostasis and pathologies. Biomed Pharmacother. 2017;94:317-25. doi:10.1016/j.biopha.2017.07.091.

71. Crowley SD, Gurley SB, Herrera MJ, Ruiz P, Griffiths R, Kumar AP, et al. Angiotensin II causes hypertension and cardiac hypertrophy through its receptors in the kidney. Proc Natl Acad Sci U S A. 2006;103:17985-90. doi:10.1073/pnas.0605545103.

72. Barreto-Chaves ML, Mello-Aires M. Effect of luminal angiotensin II and ANP on early and late cortical distal tubule HCO3- reabsorption. Am J Physiol. 1996;271:F977-84. doi:10.1152/ajprenal.1996.271.5.F977.

73. Kwon T-H, Nielsen J, Kim Y-H, Knepper MA, Frøkiaer J, Nielsen S. Regulation of sodium transporters in the thick ascending limb of rat kidney: response to angiotensin II. Am J Physiol Renal Physiol. 2003;285:F152-65. doi:10.1152/ajprenal.00307.2002.

74. Rocque BL, Babayeva S, Li J, Leung V, Nezvitsky L, Cybulsky AV, et al. Deficiency of the planar cell polarity protein Vangl2 in podocytes affects glomerular morphogenesis and increases susceptibility to injury. J Am Soc Nephrol. 2015;26:576-86. doi:10.1681/ASN.2014040340.

75. Tojo A, Tisher CC, Madsen KM. Angiotensin II regulates $\mathrm{H}(+)$-ATPase activity in rat cortical collecting duct. Am J Physiol. 1994;267:F1045-51. doi:10.1152/ajprenal.1994.267.6.F1045.

76. Xie K, Xu C, Zhang M, Wang M, Min L, Qian C, et al. Yes-associated protein regulates podocyte cell cycle re-entry and dedifferentiation in adriamycin-induced nephropathy. Cell Death Dis. 2019;10:915. doi:10.1038/s41419-019-2139-3.

77. Wang T, Giebisch G. Effects of angiotensin II on electrolyte transport in the early and late distal tubule in rat kidney. Am J Physiol. 1996;271:F143-9. doi:10.1152/ajprenal.1996.271.1.F143.

78. Faubert PF, Chou SY, Porush JG. Regulation of papillary plasma flow by angiotensin II. Kidney Int. 1987;32:472-8. doi:10.1038/ki.1987.234.

79. Hall JE. Control of sodium excretion by angiotensin II: intrarenal mechanisms and blood pressure regulation. Am J Physiol. 1986;250:R960-72. doi:10.1152/ajpregu.1986.250.6.R960.

80. Ruan X, Oliverio MI, Coffman TM, Arendshorst WJ. Renal vascular reactivity in mice: Angllinduced vasoconstriction in AT1A receptor null mice. J Am Soc Nephrol. 1999;10:2620-30.

81. Ames MK, Atkins CE, Pitt $B$. The renin-angiotensin-aldosterone system and its suppression. J Vet Intern Med. 2019;33:363-82. doi:10.1111/jvim.15454. 
82. Hayashi K, Sasamura H, Nakamura M, Azegami T, Oguchi H, Sakamaki Y, Itoh H. KLF4-dependent epigenetic remodeling modulates podocyte phenotypes and attenuates proteinuria. J Clin Invest. 2014;124:2523-37. doi:10.1172/JCI69557.

83. He W, Kang YS, Dai C, Liu Y. Blockade of Wnt/ $\beta$-catenin signaling by paricalcitol ameliorates proteinuria and kidney injury. J Am Soc Nephrol. 2011;22:90-103. doi:10.1681/ASN.2009121236.

84. Liu J, Hou W, Guan T, Tang L, Zhu X, Li Y, et al. Slit2/Robo1 signaling is involved in angiogenesis of glomerular endothelial cells exposed to a diabetic-like environment. Angiogenesis. 2018;21:23749. doi:10.1007/s10456-017-9592-3.

85. Sakurai A, Ono H, Ochi A, Matsuura M, Yoshimoto S, Kishi S, et al. Involvement of Elf3 on Smad3 activation-dependent injuries in podocytes and excretion of urinary exosome in diabetic nephropathy. PLoS One. 2019;14:e0216788. doi:10.1371/journal.pone.0216788.

86. Schwarz A, Möller-Hackbarth K, Ebarasi L, Unnersjö Jess D, Zambrano S, Blom H, et al. Coro2b, a podocyte protein downregulated in human diabetic nephropathy, is involved in the development of protamine sulphate-induced foot process effacement. Sci Rep. 2019;9:8888. doi:10.1038/s41598-019-45303-y.

87. Brand S, Amann K, Schupp N. Angiotensin II-induced hypertension dose-dependently leads to oxidative stress and DNA damage in mouse kidneys and hearts. J Hypertens. 2013;31:333-44. doi:10.1097/HJH.0b013e32835ba77e.

88. Fuchshofer R, Ullmann S, Zeilbeck LF, Baumann M, Junglas B, Tamm ER. Connective tissue growth factor modulates podocyte actin cytoskeleton and extracellular matrix synthesis and is induced in podocytes upon injury. Histochem Cell Biol. 2011;136:301-19. doi:10.1007/s00418011-0844-9.

89. Kang M-K, Park S-H, Kim Y-H, Lee E-J, Antika LD, Kim DY, et al. Chrysin ameliorates podocyte injury and slit diaphragm protein loss via inhibition of the PERK-elF2 $\alpha$-ATF-CHOP pathway in diabetic mice. Acta Pharmacol Sin. 2017;38:1129-40. doi:10.1038/aps.2017.30.

90. Qi H, Casalena G, Shi S, Yu L, Ebefors K, Sun Y, et al. Glomerular Endothelial Mitochondrial Dysfunction Is Essential and Characteristic of Diabetic Kidney Disease Susceptibility. Diabetes. 2017;66:763-78. doi:10.2337/db16-0695.

91. Zou H-H, Yang P-P, Huang T-L, Zheng X-X, Xu G-S. PLK2 Plays an Essential Role in High D-GlucoseInduced Apoptosis, ROS Generation and Inflammation in Podocytes. Sci Rep. 2017;7:4261. doi:10.1038/s41598-017-00686-8.

92. Stankewich MC, Moeckel GW, Ji L, Ardito T, Morrow JS. Isoforms of Spectrin and Ankyrin Reflect the Functional Topography of the Mouse Kidney. PLoS One. 2016;11:e0142687. doi:10.1371/journal.pone.0142687.

93. Fu $Y$, Wang $C$, Zhang $D$, Chu $X$, Zhang $Y$, Li J. miR-15b-5p ameliorated high glucose-induced podocyte injury through repressing apoptosis, oxidative stress, and inflammatory responses by targeting Sema3A. J Cell Physiol. 2019;234:20869-78. doi:10.1002/jcp.28691.

94. Aggarwal PK, Veron D, Thomas DB, Siegel D, Moeckel G, Kashgarian M, Tufro A. Semaphorin3a promotes advanced diabetic nephropathy. Diabetes. 2015;64:1743-59. doi:10.2337/db14-0719. 
A

Proliferation Medium

Adv. RPMI + Retinoic acid + low density
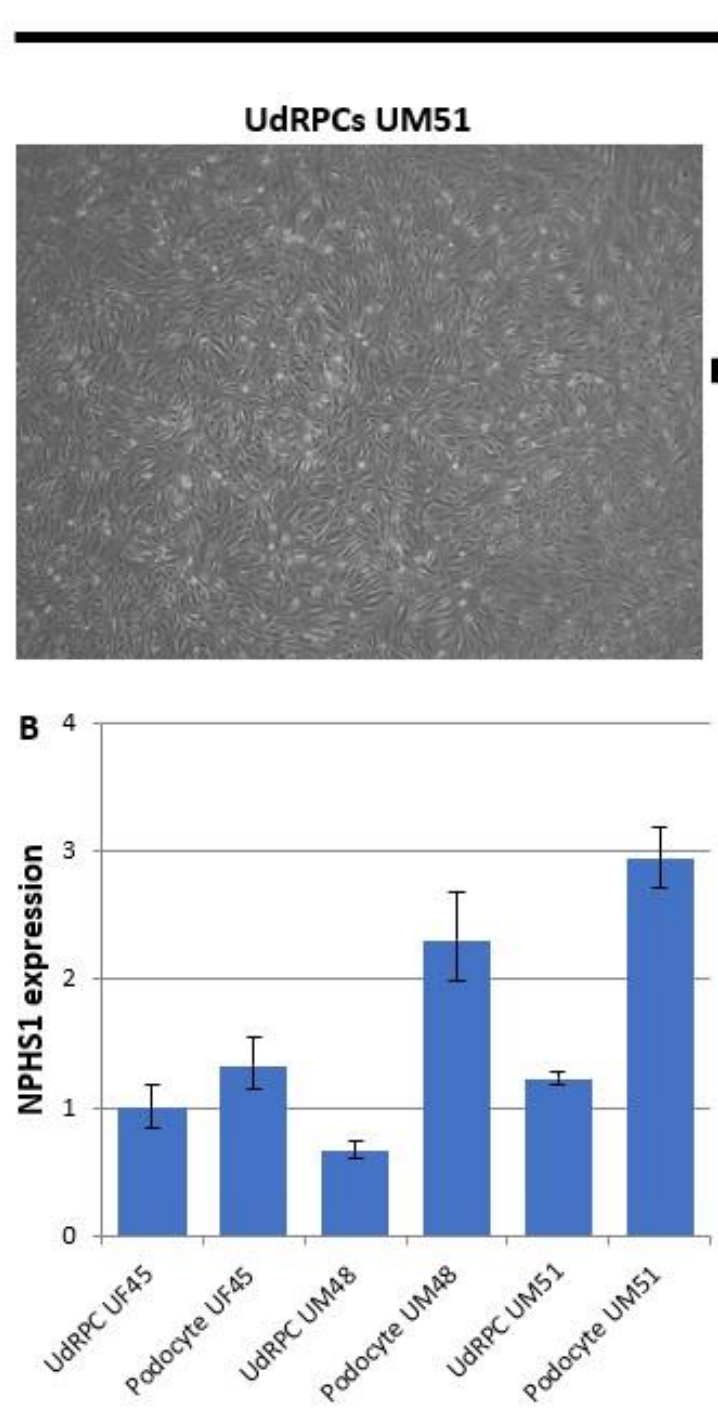

D WT1

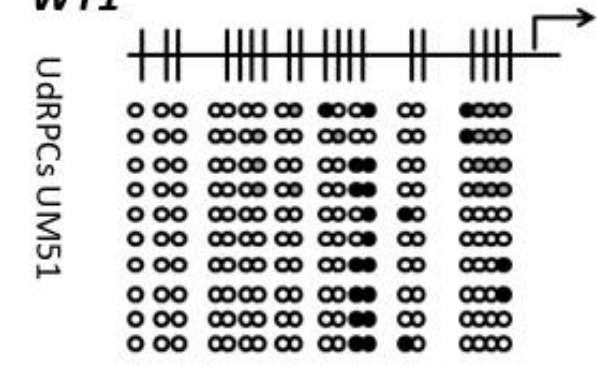

Differentiation
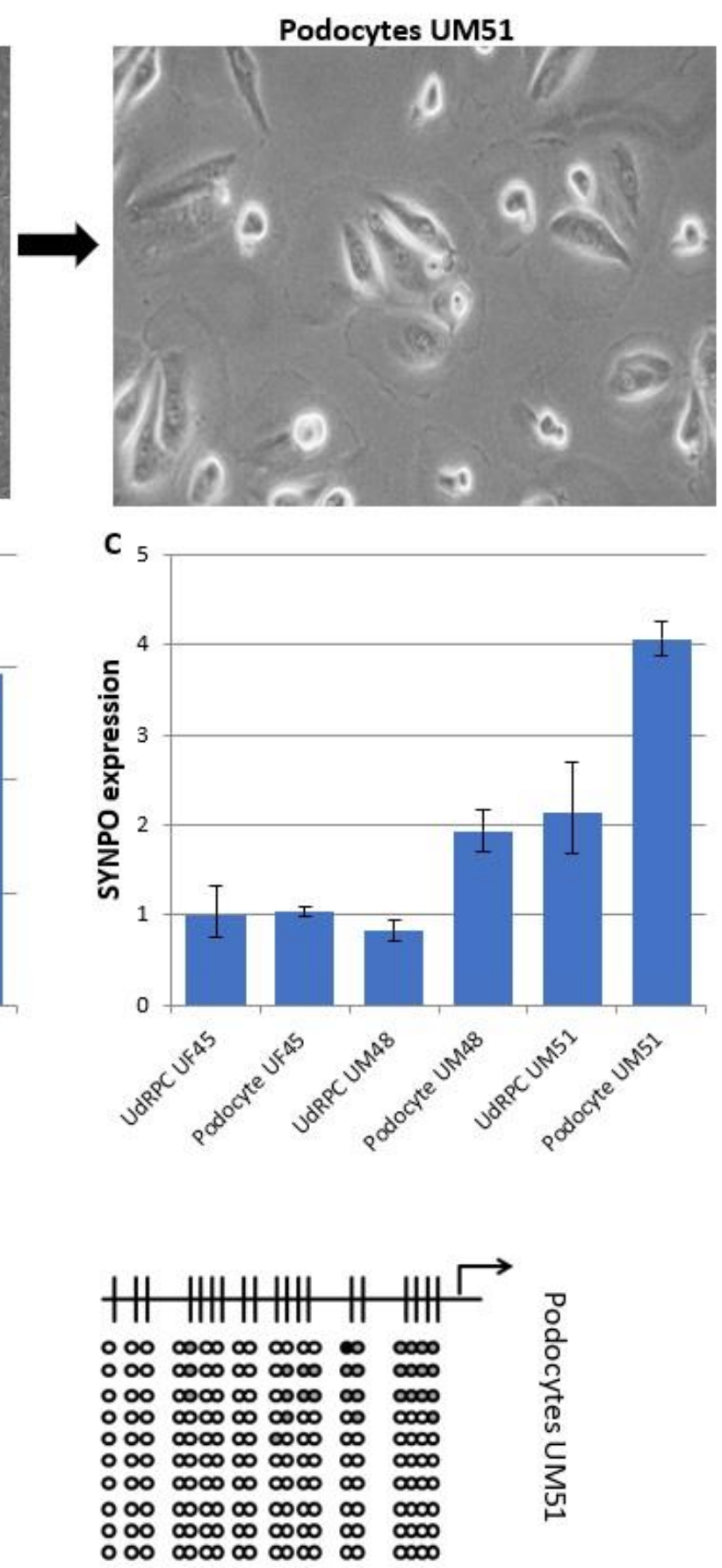
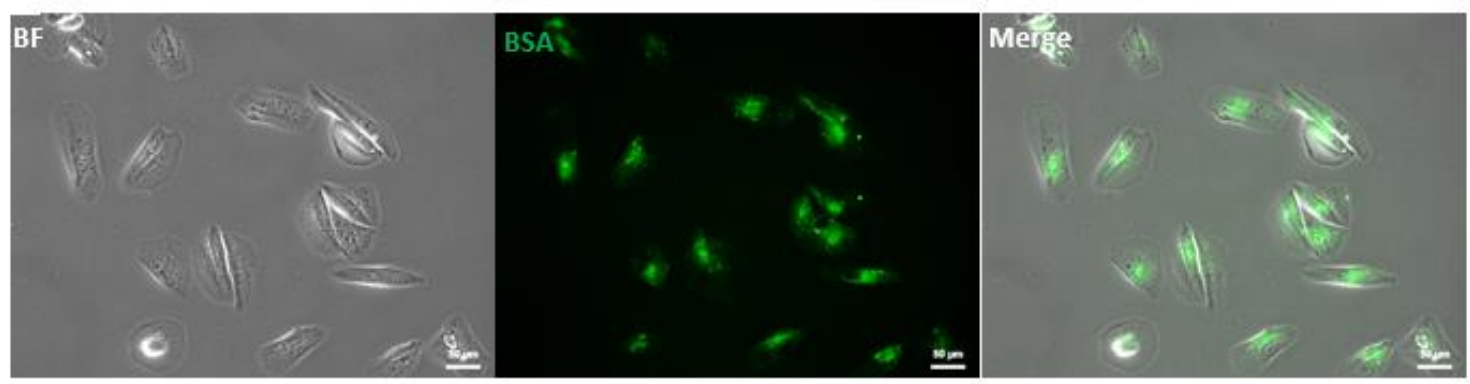
bioRxiv preprint doi: https://doi.org/10.1101/2021.03.18.436037; this version posted March 18, 2021. The copyright holder for this preprint

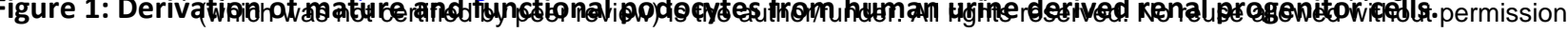

The self-renewal capacity of UdRPCs is maintained by proliferation medium. Differentiation of UdRPCs into podocytes is induced by low density cultivation in adv. RPMI supplemented with $30 \mu \mathrm{M}$ retinoic acid (a). Expression of the podocyte associated genes NPHS1 (b) and SYNPO (c) was determined by quantitative real time PCR. Bisulfite genomic sequencing of a $270 \mathrm{bp}$ long WT1 promoter fragment, spanning 19 CpG-dinucleotides, provide detailed information about the dynamic DNA Methylation changes occurring during the differentiation process into podocytes (d). Black, white and grey circles refer to methylated, unmethylated and undefined CpG dinucleotides, respectively. UdRPCs show dense methylation patterns at CpG position 12 and 13, whilst differentiated podocytes show a lack of methylation. Infiltration of exogenous BSA supplemented into the culture medium confirmed endocytosis of Albumin in UM51 podocytes (e). 

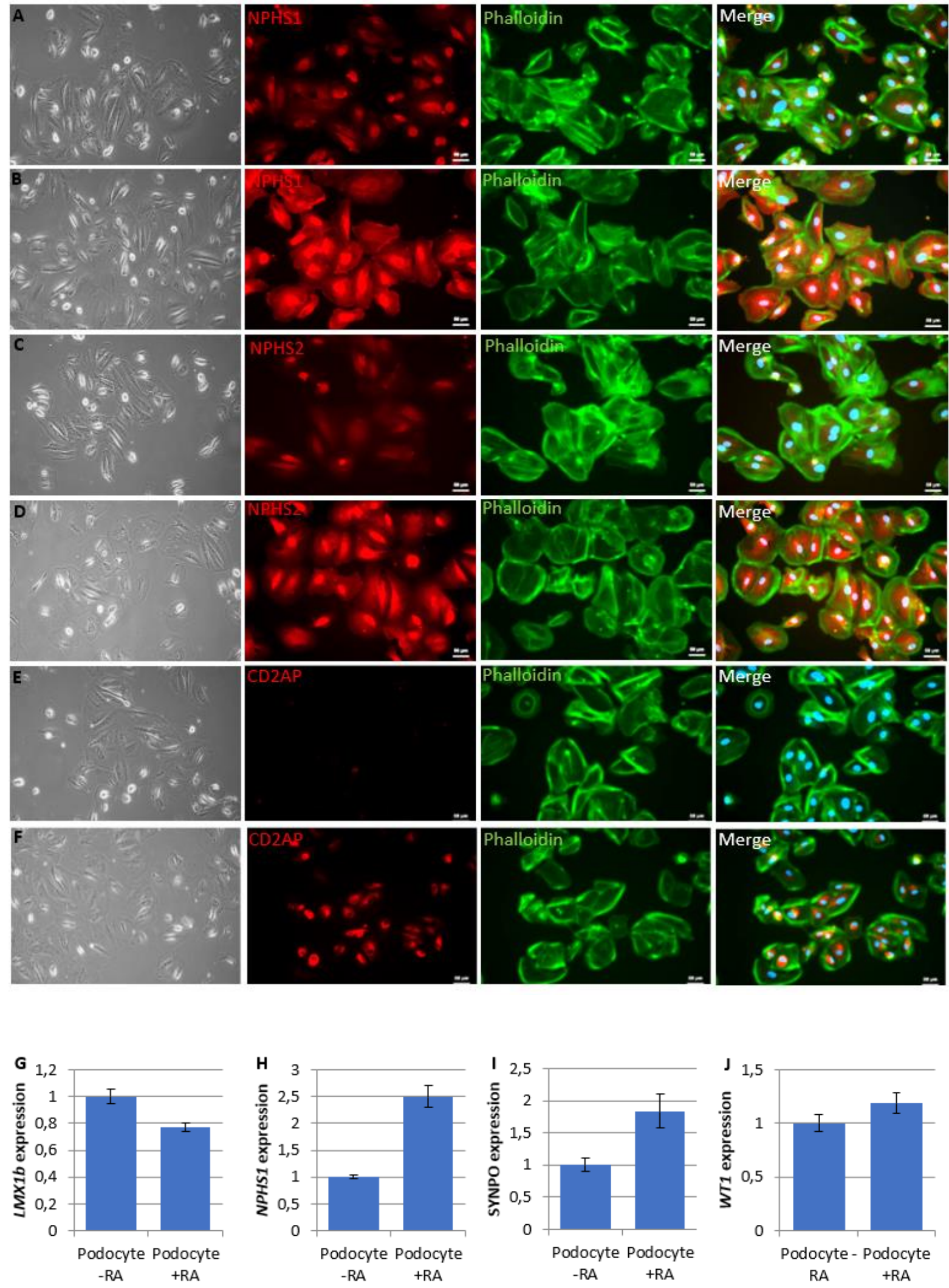

Figure 2: Retinoic acid enhances the maturation of UdRPC-derived podocytes.

UdRPCs differentiated into podocytes, by low density cultivation in advanced RPMI medium supplemented without (a, $c$, e) and with (b, d, f) $30 \mu \mathrm{M}$ retinoic acid. Immunofluorescence-based detection of NPHS1 (a, b), NPHS2 (c, d) and CD2AP (e, f) expression. Podocyte cytoskeleton was stained with phalloidin. Expression of podocyte markers LMX1b (g), NPHS1 (h), SYNPO (i), and WT1 (j) was determined by quantitative real time PCR. 
A

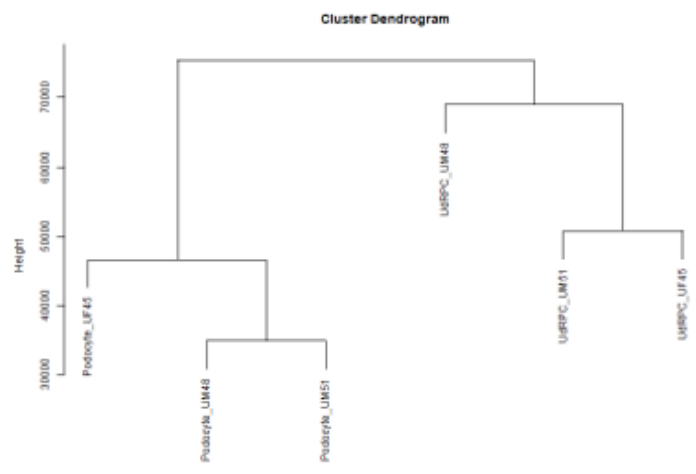

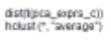

B

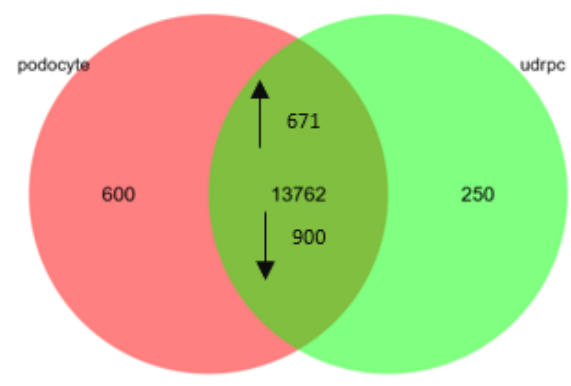

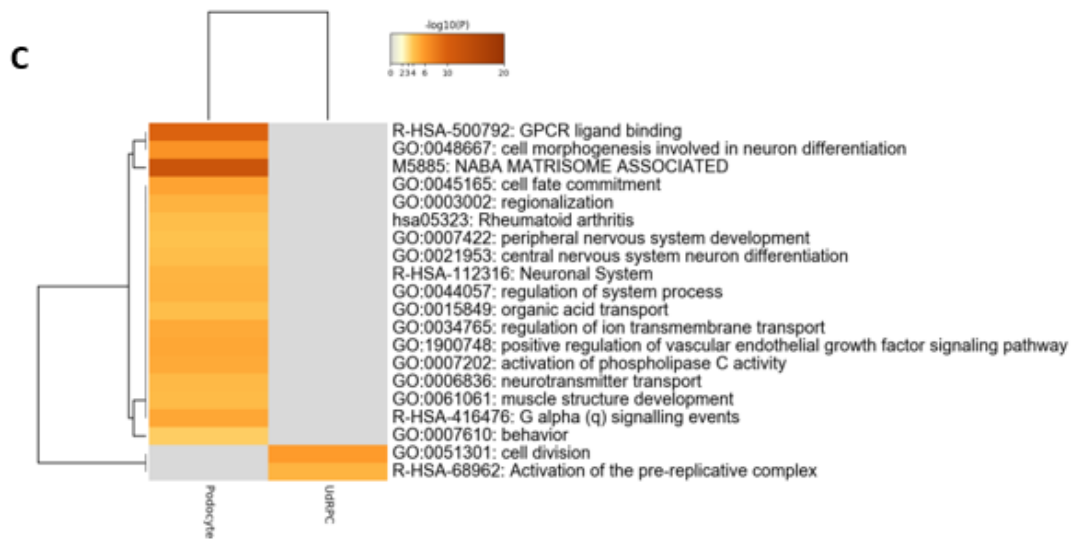

D

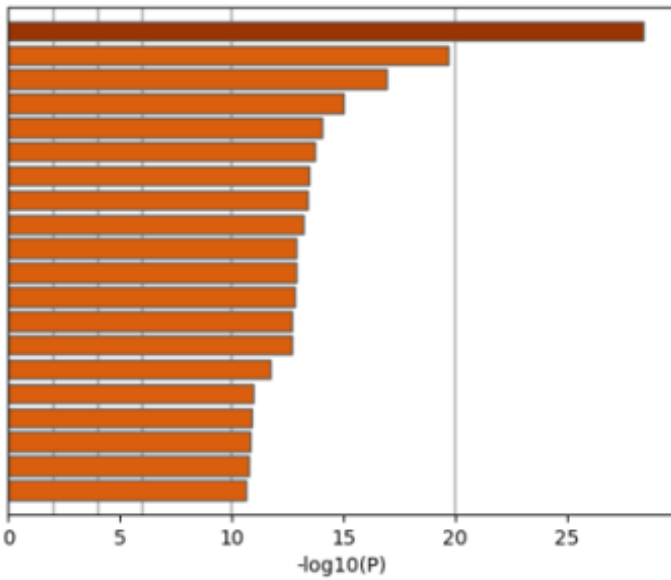

GO:0030155: regulation of cell adhesion

hsa04668: TNF signaling pathway
GO:0030335: positive regulation of cell migration

GO:0030198: extracellular matrix organization

GO:0034612: response to tumor necrosis factor

GO:0031589: cell-substrate adhesion

GO:0009611: response to wounding

GO:0010942: positive regulation of cell death

GO:0009615: response to virus

GO:0002009: morphogenesis of an epithelium

GO:0001817: regulation of cytokine production

R-HSA-5660526: Response to metal ions

hsa04510: Focal adhesion

GO:0070848: response to growth factor

hsa05164: Influenza A

GO:0040008: regulation of growth

M5885: NABA MATRISOME ASSOCIATED

GO:0032496: response to lipopolysaccharide

GO:0070555: response to interleukin-1

$\mathbf{E}$

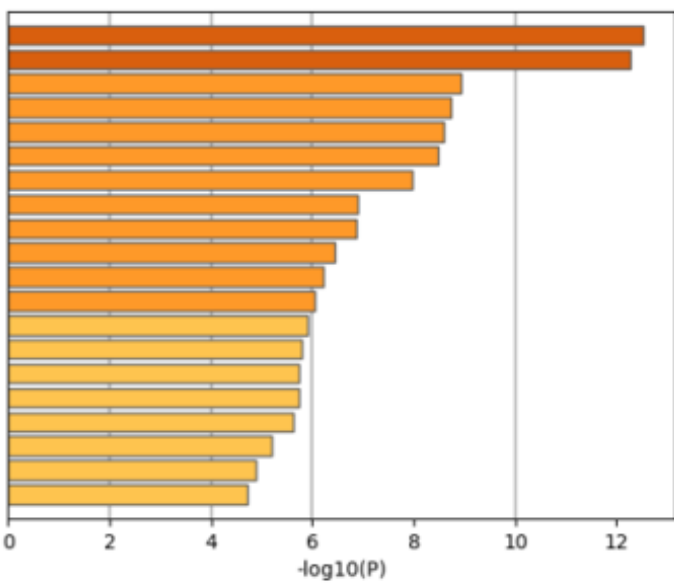

GO:0060271: cilium assembly

GO:0051186: cofactor metabolic process

hsa01212: Fatty acid metabolism

GO:0010564: regulation of cell cycle process

GO:0007017: microtubule-based process

GO:0016569: covalent chromatin modification

GO:0007389: pattern specification process

R-HSA-196849: Metabolism of water-soluble vitamins and cofactors

R-HSA-556833: Metabolism of lipids

GO:0097711: ciliary basal body-plasma membrane docking

GO:1905515: non-motile cilium assembly

GO:0032259: methylation

M121: PID MTOR 4PATHWAY

hsa00640: Propanoate metabolism

CORUM:164: Cohesin-SA1 complex

GO:0018916: nitrobenzene metabolic process

GO:1902115: regulation of organelle assembly

R-HSA-2990846: SUMOylation

GO:0006281: DNA repair

GO:0051056: regulation of small GTPase mediated signal transduction 
Figure 3: Comparative transcriptome and Gene Ontology analysis of urine-derived renal progenitors UF45, UM 48, UM51 and derived podocytes.

A hierarchical cluster dendrogram revealed distinct clusters of UdRPCs and their derived podocytes. (A). Expressed genes (det-p < 0.05$)$ in UdRPCs and podocytes compared in the Venn diagram (B), shows distinct (600 in podocytes; 200 in UdRPCs) and overlapping (13762) gene expression patterns. Of the overlapping genes, 671 are upregulated and 900 downregulated in podocytes. The most over- represented GO BP-terms exclusive in either UdRPCs or podocytes are shown in c and including cell division and activation of the pre replicative complex for the UdRPCs and cell fate commitment, cell morphogenesis and regulation of ion transport for the podocytes. The upregulated 671 genes in podocytes, are associated with cell adhesion, positive regulation of cell migration, cell substrate adhesion, morphogenesis of an epithelium and regulation of cytokine production, while downregulated 900 genes are associated with cell cycle processes and methylation (E). 
A
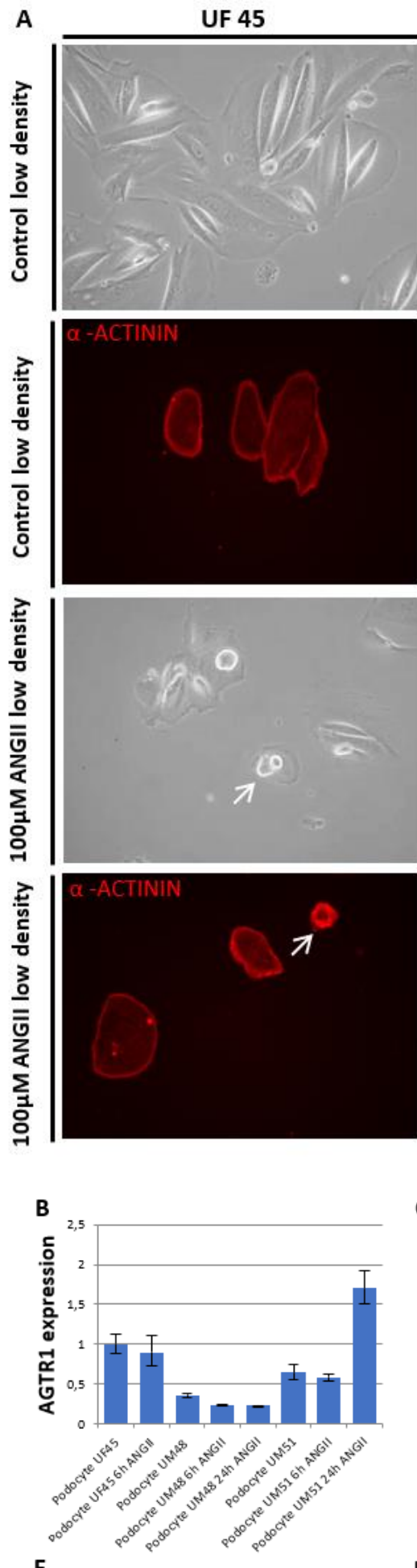

E

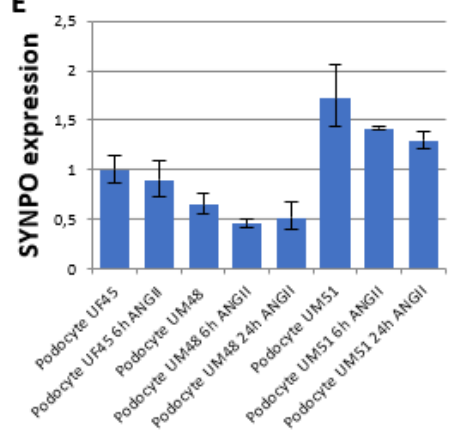

UM 48
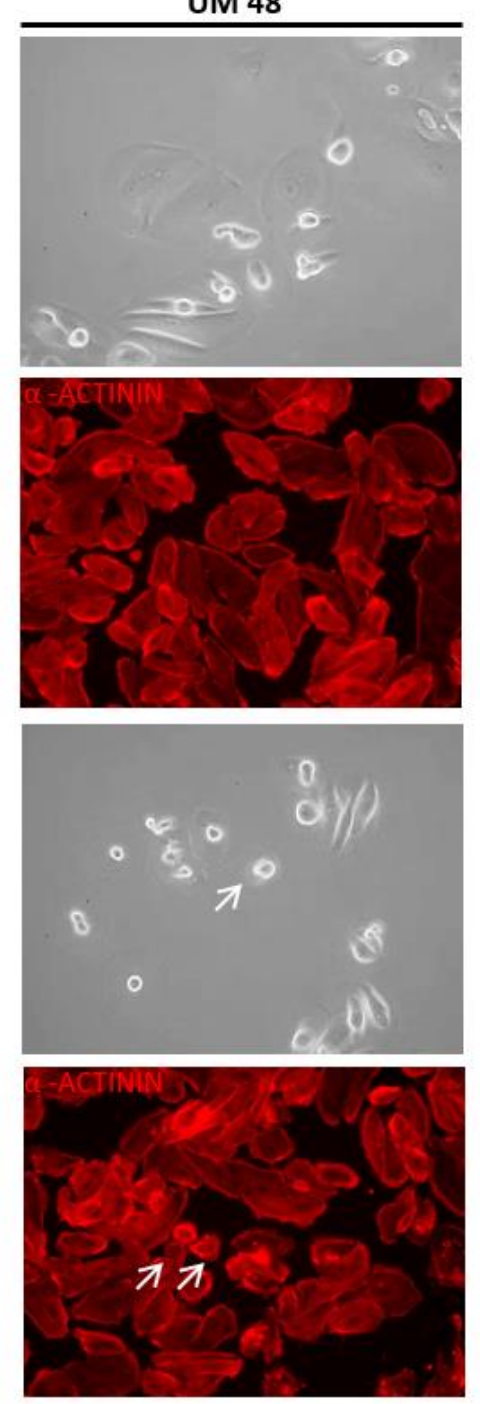

C

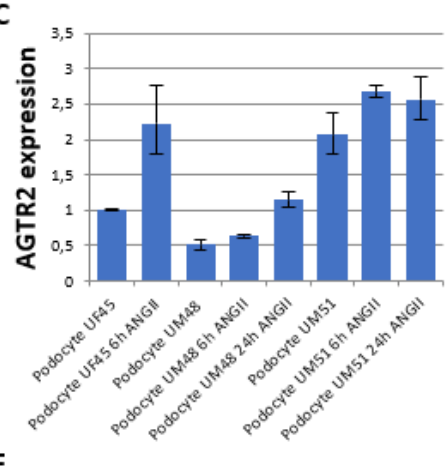

F

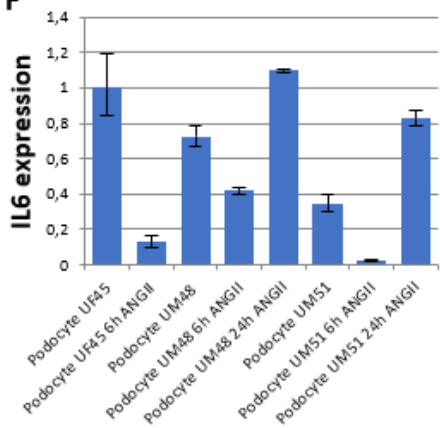

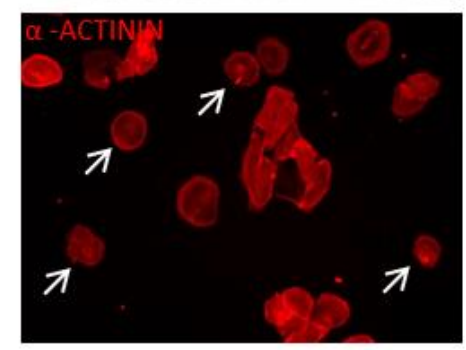
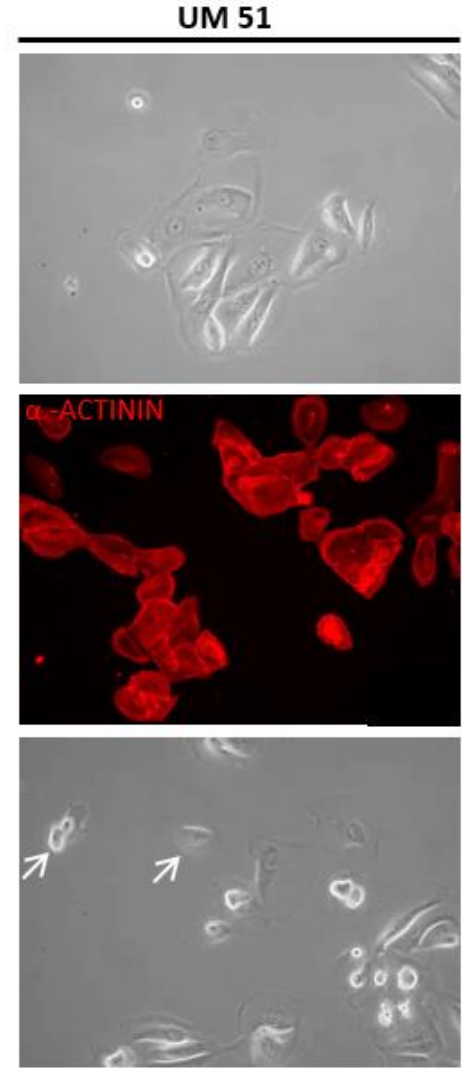

D

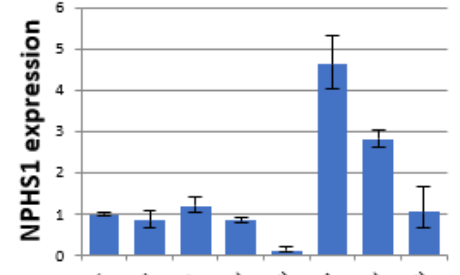

G

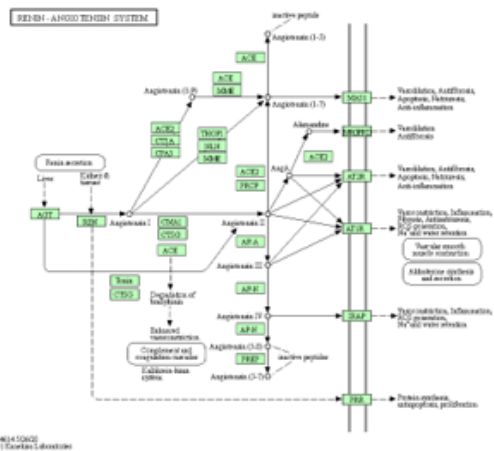




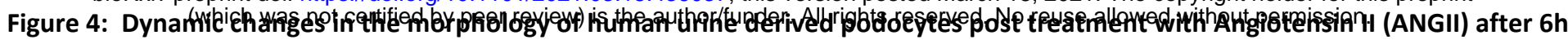
and $24 \mathrm{~h}$.

UdRPC-differentiated podocytes from UF45, UM48 and UM51. The top panel (phase contrast) shows the typical "fried egg“ shaped podocyte morphology. The lower two panels show morphology changes after $6 \mathrm{~h}$ of $100 \mu \mathrm{M}$ ANGII treatment. Podocyte cytoskeleton was visualized by immunofluorescence-based detection of $\alpha$-ACTININ in red (a). ANG II interferes with the cytoskeleton of the podocytes, inhibiting podocyte spreading and resulting in the loss of foot processes and the observed roundish phenotype (indicated by the white arrows). Expression of ANGII receptors AGTR1 (b), AGTR2 (c), expression podocyte markers NPHS1 (d), SYNPO (e) and pro inflammatory cytokine $I L-6$ (f) were determined by quantitative real time PCR normalized with the ribosomal encoding gene-RPL0. An overview of the KEGG annotated renin-angiotensin system is given in figure $4 \mathrm{~g}$. 
bioRxiv preprint doi: https://doi.org/10.1101/2021.03.18.436037; this version posted March 18, 2021. The copyright holder for this preprint

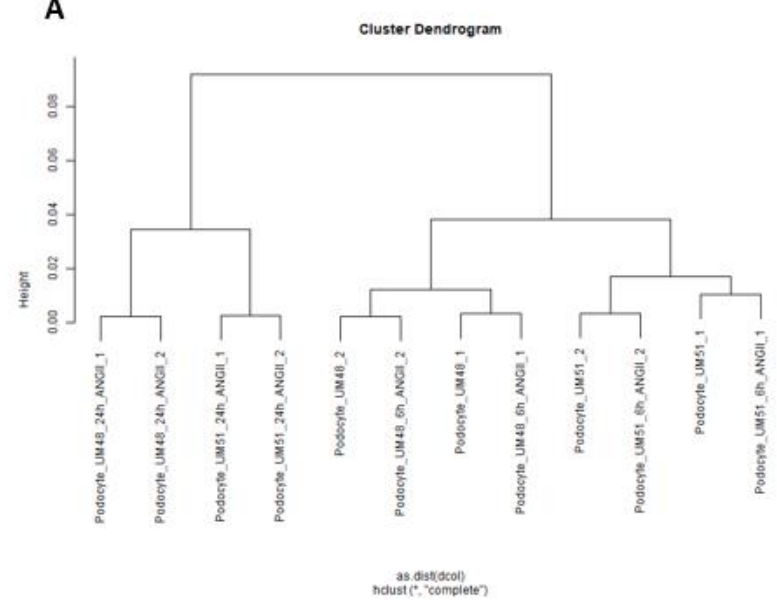

D

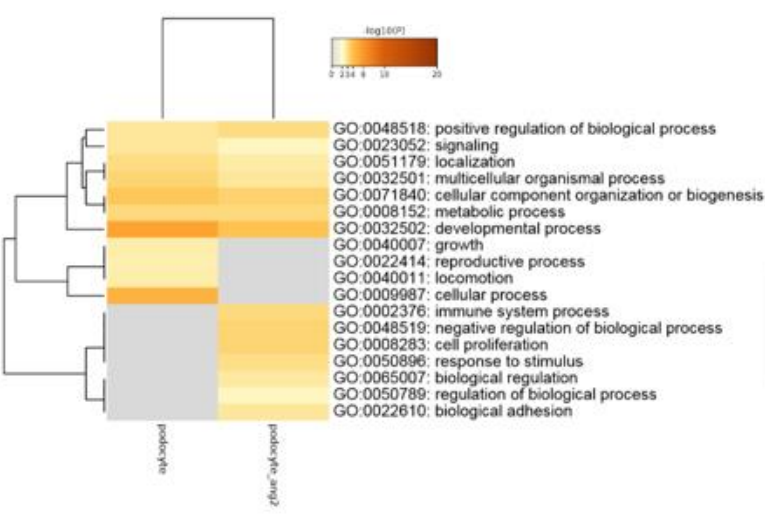

$\mathbf{F}$
B

C

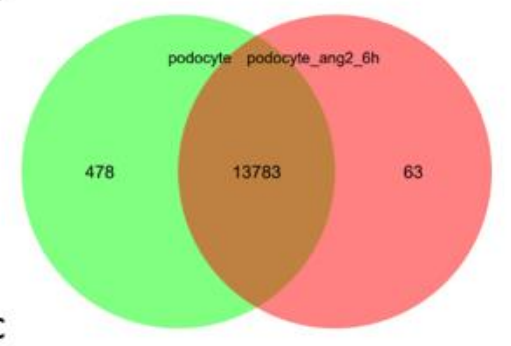

E
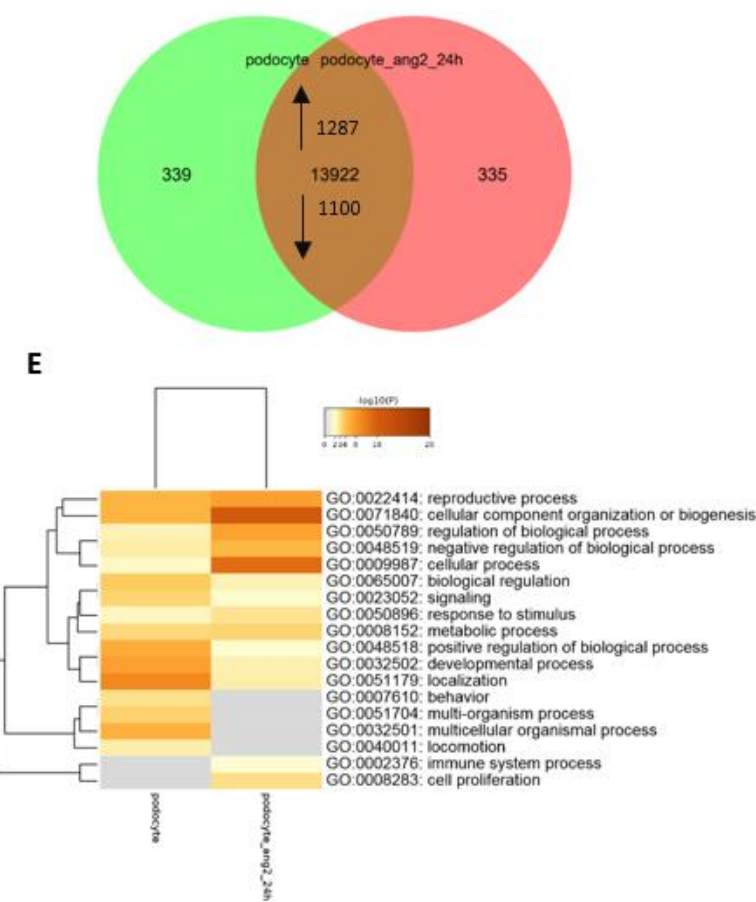

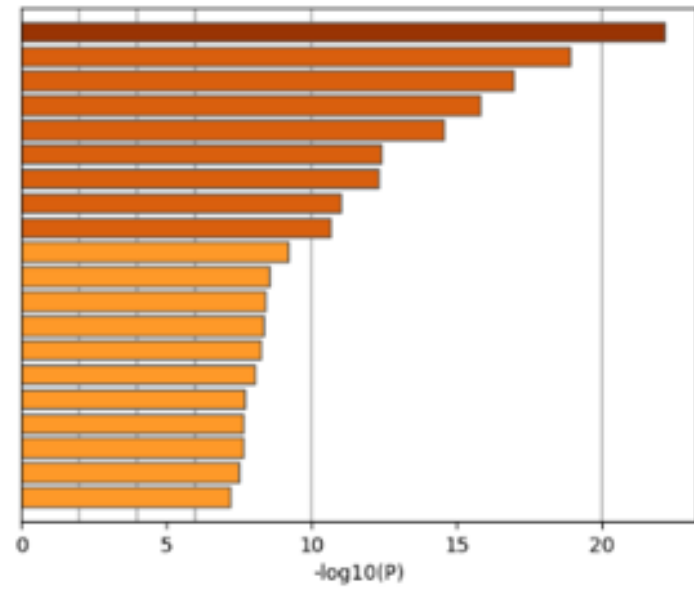

R-HSA-1640170: Cell Cycle R-HSA-8953854: Metabolism of RNA GO:0006281: DNA repair R-HSA-2990846: SUMOylation GO:0007017: microtubule-based process GO:0048285: organelle fission GO:0044770: cell cycle phase transition GO:0006397: mRNA processing GO:0032259: methylation GO:0032259: methylation GO:0006260: DNA replication GO:0009451: RNA modification GO:0051170: import into nucleus 00:0042594: response to starvation GO:0071103: DNA conformation change GO:0019439: aromatic compound catabolic process GO:0033044: regulation of chromosome organization GO:0051052: regulation of DNA metabolic process GO:0120031: plasma membrane bounded cell projection assembly GO:0061448: connective tissue development

G

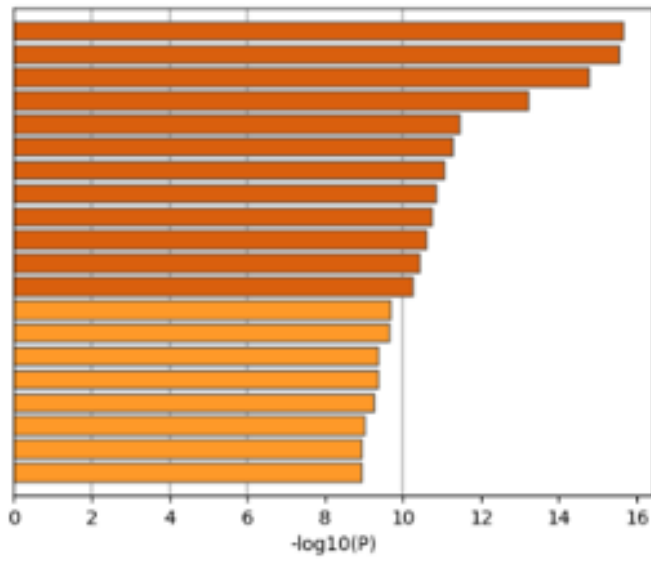

GO:0030155: regulation of cell adhesion

GO:0051272: positive regulation of cellular component movement GO:0001817: regulation of cytokine production

GO:0009611: response to wounding

G0:0051403: stress-activated MAPK cascade

GO:0007249: I-kappaB kinase/NF-kappaB signaling

hsa04668: TNF signaling pathway

G0:0034330: cell junction organization

Go:0007409: axonogenesis

Go:0008544: epidermis development

60:0051092: positive regulation of NF-kappaB transcription factor activity

60:0051345: positive regulation of hydrolase activity

60:0022612: gland morphœgenesis

0:0048729: tissue morphogenesis

-HSA-446728: Cell junction organization

Go:0019221: cytokine-mediated signaling pathway

hs:005168: Herpes simplex infection

60:0002064: epithelial cell development

00:0030036: actin cytoskeleton organization 

(which was not certified by peer review) is the author/funder. All rights reserved. No reuse allowed withoutpermission.
Figure 5: Comparative transcriptome and Gene Ontology analysis of untreated podocytes UM48 and UM51 and treated with Angiotensin II for $6 \mathrm{~h}$ and $24 \mathrm{~h}$.

Podocytes were treated with ANGII $(100 \mu \mathrm{M})$ for $6 \mathrm{~h}$ and $24 \mathrm{~h}$. The hierarchical cluster dendrogram revealed 2 distinct clusters- $24 \mathrm{~h}$ treated and $6 \mathrm{~h}$ treated and untreated cells as a cluster. (a). Expressed genes (det-p $<0.05$ ) in podocytes and after ANGII treatment are compared by Venn diagrams after $6 \mathrm{~h}$ (b) and $24 \mathrm{~h}$ (c), revealing unique and overlapping expression patterns. The most overrepresented GO BP-terms (13922 genes) common in either condition are shown in d after $6 \mathrm{~h}$ of treatment and e after $24 \mathrm{~h}$. The terms include locomotion, growth, immune system process, response to stimulus biological adhesion, metabolic process, and cell proliferation for the treated podocytes. The 10 most over-represented GO BP-terms in the up (1287) and down (1100)-regulated genes in podocytes treated for $24 \mathrm{~h}$ with ANGII in comparison to untreated cells are shown in (f). The upregulated genes are associated with cell cycle, DNA repair, cell cycle phase transition, methylation and regulation of cellular response to stress while downregulated genes are associated with regulation of cell adhesion, cell junction organization, axonogenesis, actin cytoskeleton organization and tissue and gland morphogenesis (g). 


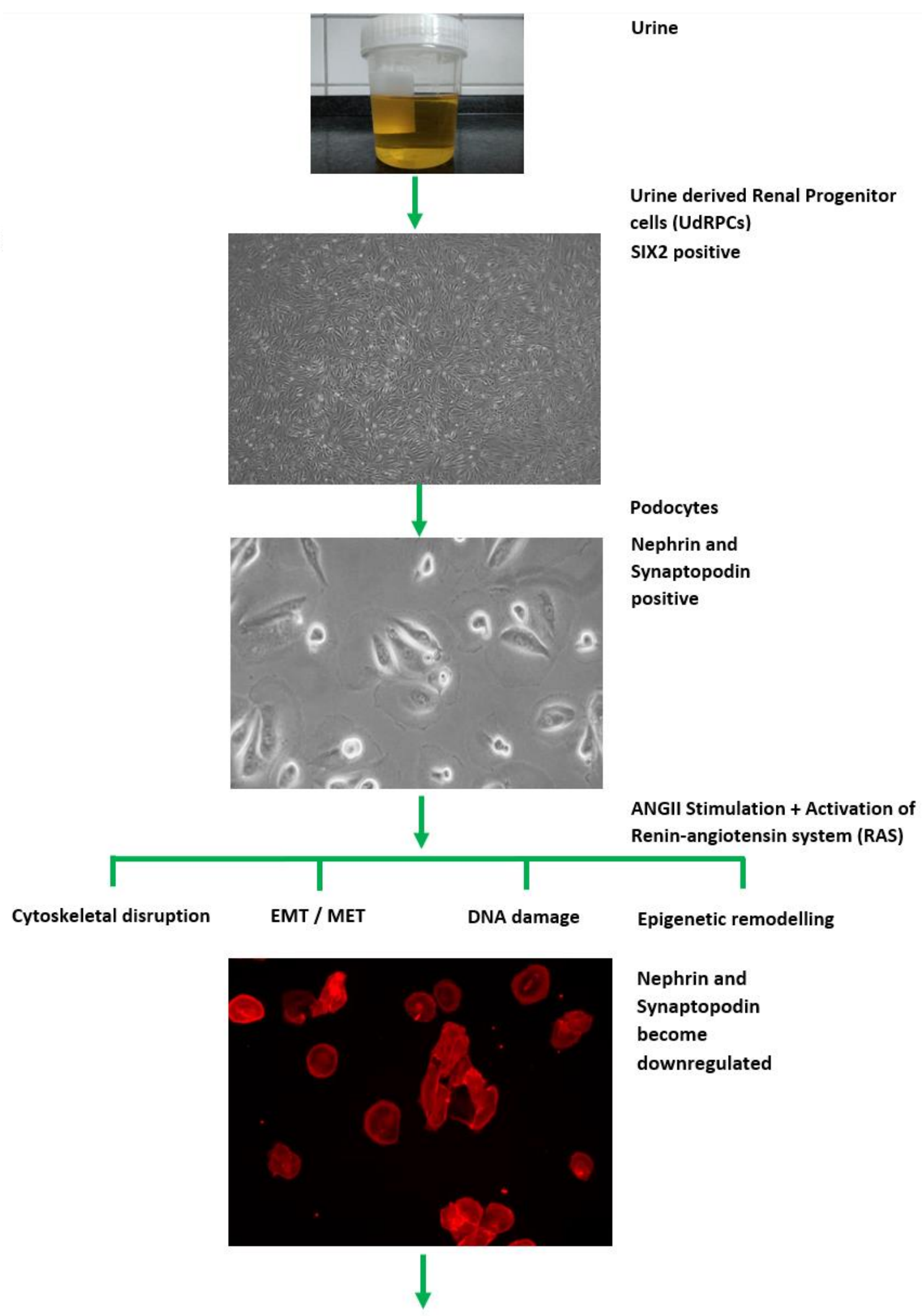

Subsequent podocyte damage leading to chronic kidney disease

Figure 6: Graphical summary of this study.

SIX2-positive renal progenitor cells were isolated directly from human urine and cultured in proliferation medium. UdRPCs differentiated into podocytes, by low density cultivation in advanced RPMI medium supplemented with $30 \mu \mathrm{M}$ retinoic acid. Stimulation with $100 \mu \mathrm{M}$ ANGII results in cytoskeletal remodelling. Furthermore, the transcriptome revealed altered expression of genes associated with epigenetic remodelling, DNA damage response, EMT and MET. ANGIl activated the renin-angiotensin system resulting in podocyte damage, -loss and ultimately leading to chronic kidney disease. 